\title{
Testing modified gravity with cosmic shear
}

\author{
J. Harnois-Déraps, ${ }^{1 \star}$ D. Munshi, ${ }^{2}$ P. Valageas,,${ }^{3,4}$ L. van Waerbeke, ${ }^{1}$ P. Brax,${ }^{3,4}$ \\ P. Coles ${ }^{2}$ and L. Rizzo ${ }^{3,4}$ \\ ${ }^{1}$ Department of Physics and Astronomy, University of British Columbia, 6224 Agricultural Road, Vancouver, B.C. V6T 1Z1, Canada \\ ${ }^{2}$ Astronomy Centre, School of Mathematical and Physical Sciences, University of Sussex, Brighton BN1 9QH, UK \\ ${ }^{3}$ CEA, IPhT, F-91191 Gif-sur-Yevette, Cedex, France \\ ${ }^{4}$ CNRS, URA, 2306, F-91191 Gif-sur-Yevette, Cedex, France
}

Accepted 2015 September 10. Received 2015 September 9; in original form 2015 June 20

\begin{abstract}
We use the cosmic shear data from the Canada-France-Hawaii Telescope Lensing Survey to place constraints on $f(R)$ and Generalized Dilaton models of modified gravity. This is highly complementary to other probes since the constraints mainly come from the non-linear scales: maximal deviations with respects to the General Relativity (GR) $+\Lambda$ cold dark matter $(\Lambda \mathrm{CDM})$ scenario occurs at $k \sim 1 h \mathrm{Mpc}^{-1}$. At these scales, it becomes necessary to account for known degeneracies with baryon feedback and massive neutrinos, hence we place constraints jointly on these three physical effects. To achieve this, we formulate these modified gravity theories within a common tomographic parametrization, we compute their impact on the clustering properties relative to a GR universe, and propagate the observed modifications into the weak lensing $\xi_{ \pm}$quantity. Confronted against the cosmic shear data, we reject the $f(R)\left\{\left|f_{R_{0}}\right|=10^{-4}, n=1\right\}$ model with more than 99.9 per cent confidence interval (CI) when assuming a $\Lambda \mathrm{CDM}$ dark matter only model. In the presence of baryonic feedback processes and massive neutrinos with total mass up to $0.2 \mathrm{eV}$, the model is disfavoured with at least 94 per cent $\mathrm{CI}$ in all different combinations studied. Constraints on the $\left\{\left|f_{R_{0}}\right|=10^{-4}, n=2\right\}$ model are weaker, but nevertheless disfavoured with at least 89 per cent CI. We identify several specific combinations of neutrino mass, baryon feedback and $f(R)$ or Dilaton gravity models that are excluded by the current cosmic shear data. Notably, universes with three massless neutrinos and no baryon feedback are strongly disfavoured in all modified gravity scenarios studied. These results indicate that competitive constraints may be achieved with future cosmic shear data.
\end{abstract}

Key words: gravitation - gravitational lensing: weak - methods: analytical - methods: numerical-methods: statistical-large-scale structure of Universe.

\section{INTRODUCTION}

Explaining the late-time acceleration of the Universe first reported in Riess et al. (1998) and Perlmutter et al. (1999) represents a major challenge in modern cosmology, and current interpretations mostly rely on the inclusion of dark energy components and/or modifications to the theory of General Relativity (GR). One important difficulty encountered in solving this puzzle relates to the fact that, by construction, the background dynamics in viable dark energy and modified gravity models are almost indistinguishable (Bertschinger 2006; Song, Hu \& Sawicki 2007a; Brax et al. 2008). These two frameworks only really decouple when considering the evolution of matter density fluctuations and of perturbations associated with the metric. In addition, there are various ways in which a modification

${ }^{\star}$ E-mail: jharno@phas.ubc.ca of gravity on large scales could account for the apparent acceleration (Clifton et al. 2013; Joyce et al. 2015). Exploiting this, many observational probes based on large-scale structure formation have been proposed to test theories of modified gravity, including galaxy clustering (Oyazu, Lima \& Hu 2008; Pogosian \& Silvestri 2008), integrated Sachs-Wolfe (ISW) effect in the cosmic microwave background (CMB) anisotropies and its cross-correlation with galaxy density (Song, Peiris \& Hu 2007b), cluster abundance (Jain \& Zhang 2008; Lombriser et al. 2010), peculiar velocities (Li et al. 2013a; Johnson et al. 2014, 2015), redshift-space distortions from spectroscopic surveys (Guzzo et al. 2008; Jennings et al. 2012; Asaba et al. 2013), $21 \mathrm{~cm}$ observations (Hall, Bonvin \& Challinor 2013) and weak gravitation lensing (Heavens, Kitching \& Verde 2007; Schimdt 2008; Tsujikawa \& Tatekawa 2008; Simpson et al. 2013; Wilcox et al. 2015). Recent results from the Planck Collaboration XIV (2015) combine data from the CMB temperature and lensing maps with other low-redshift probes and provide constraints 
on coupled dark energy scenarios, $k$-essence and $f(R)$ models. As explained therein, the effects of modified gravity on the CMB can affect both the largest scales - via the ISW effect or modifications to the lensing potential for instance - and small scales - i.e. via SZ effects. However, these measurements are restricted in angular range ( $\ell<200$ for ISW and $\ell<2048$ for lensing maps), and are unfortunately not precise enough to single-out a preferred model. Combinations with other independent measurements are therefore highly desirable.

In this paper, we investigate the extent to which current weak lensing surveys can constrain departures from GR. In particular, we study the signatures of two specific classes of parametrized modified gravity theories, the $f(R)$ and the Generalized Dilaton models (see Joyce et al. 2015 for a review), on the cosmic shear measurement extracted from the Canada-France-Hawaii Telescope Lensing Survey (CFHTLenS; Erben et al. 2013). These models are known to cause an enhancement of structure formation over scales in the range [0.2-20] $\mathrm{Mpc} h^{-1}$, an effect which could be detectable with current lensing surveys. In addition, the departure of these models from GR is maximal at scales of $k \sim 1 h^{-1} \mathrm{Mpc}$, which are difficult to interpret with other clustering data due to the large uncertainty in the galaxy bias. This makes the weak lensing approach special, probing modified gravity models at the scale of influence of the 'fifth' force. In that sense, it is very much complementary to the CMB measurement (Planck Collaboration XIV 2015) as it provides a strong handle on the small scales. Note that the Planck analysis includes the cosmic shear measurement from the CFHTLenS data (Heymans et al. 2013; Kilbinger et al. 2013), but excludes all of the data affected by non-linear scales, in contrast with this paper.

In its approach, this paper is an extension of Harnois-Déraps, van Waerbeke, Viola \& Heymans (2015, hereafter HWVH), where the same data were used to place joint constraints on baryon feedback models and on the sum of neutrino mass. The general idea can be understood as follows: on the one hand, the accuracy achieved by modern CMB experiments (Hinshaw et al. 2013; Planck Collaboration XIII 2015) on most $\Lambda$ cold dark matter $(\Lambda \mathrm{CDM})$ parameters is at the percent level; on the other hand, the modified gravity effects we are looking for affect the baseline signal by up to 20 per cent at small scales. It is therefore justified to assume a fixed cosmology and search for possible deviations. Any residual uncertainty in the cosmology can thereafter be treated as systematic uncertainty in the analysis. While next-generation weak lensing experiments such as RCSLenS, ${ }^{1}$ DES, ${ }^{2}$ KiDS, ${ }^{3}$ Euclid $^{4}$ and LSST $^{5}$ will have enough statistical power to repeat this analysis in a full MCMC pipeline, we demonstrate here that we can find interesting results with simpler tools and existing data.

This paper is organized as follows: in Section 2, we review the theoretical formulation of structure formation in $f(R)$ and Dilaton gravity theories; Section 3 describes the theoretical and numerical modelling of the weak lensing signal, and details our cosmic shear measurement from the CFHTLenS data. In Section 4, we present and discuss our results, and conclude in Section 5. The baseline cosmological parameters that are used throughout our study correspond to the WMAP $9+\mathrm{BAO}+\mathrm{SN} \Lambda \mathrm{CDM}$ cosmology: $h=0.6898, \Omega_{\mathrm{m}}$ $=0.2905, \Omega_{\Lambda}=0.7095, \Omega_{\mathrm{K}}=0, w=-1, \sigma_{8}=0.831$ and $n_{\mathrm{s}}$

\footnotetext{
${ }^{1}$ http://www.rcslens.org

2 http://www.darkenergysurvey.org

3 http://kids.strw.leidenuniv.nl

${ }^{4}$ http://sci.esa.int/euclid

${ }^{5}$ http://www.lsst.org/lsst
}

$=0.969$. The reason why we did not opt for the Planck cosmology is to minimize the effect of the known cosmological tension in our model rejection strategy. Otherwise, this would involve a full MCMC calculation including all cosmological parameters and both data sets as in MacCrann et al. (2014), which is not necessary in our approach. In the end however, we do marginalize over this cosmological discrepancy.

\section{MODIFIED GRAVITY THEORIES}

Modified theories of gravity can be distinguished by their screening properties in dense environments. Indeed, given the strong Solar system constraints, these theories need to have a built-in screening mechanism, suppressing the deviations from GR. ${ }^{6}$ Three types of such mechanisms have emerged in the last few years: the Chameleon, K-mouflage and Vainshtein models (see Brax \& Valageas 2014a for a comparison between these different screening mechanisms). On the one hand, K-mouflage and Vainshtein models involve non-linear kinetic terms describing additional scalar fields whose presence modifies GR predictions. On the other hand, modifications of the Chameleon type can be broadly categorized as either containing additional couplings between the metric and new scalar fields, or involving extra geometric terms. These two equivalent descriptions can be captured by the tomographic parametrization, which will be used throughout this paper (Brax, Davis \& Li 2012b,c).

In all Chameleon cases, modifications of gravity induce a global enhancement of the effective force of gravity, due to the 'fifth force', which directly translates into an increase of structure formation. A decrease of the clustering rate on small scales can be achieved for certain Galileon models subject to the Vainshtein screening mechanism (Li et al. 2013b), as the effective Newton constant becomes less than $\mathcal{G}_{\mathrm{N}}$ in this case. These theories do not belong to the class of models studied here as they are characterized by the Vainshtein mechanism when the $f(R)$ and Generalized Dilaton models obey the chameleon mechanism.

In this section, we review two different types of modified gravity, namely the Dilaton and the $f(R)$ models; we describe their distinct screening mechanisms, and detail their parametrization in the context of large-scale structure formation.

\subsection{Gravity in Dilaton models}

The Dilaton and Symmetron ${ }^{7}$ theories of modified gravity are Chameleon models that exhibit the Damour-Polyakov property (Damour \& Polyakov 1994), according to which the coupling between the scalar field $\varphi$ and the rest of the matter components approaches zero in dense environments (Pietroni 2005; Olive \& Pospelov 2008; Hinterbichler \& Khoury 2010). In contrast to the case of $f(R)$ theories, described in Section 2.2 below, the scalar field here takes on a small mass everywhere and thus mediates a long-range (screened) force.

\footnotetext{
${ }^{6}$ Note that modified gravity models that do not couple with the baryon sector do not necessarily need screening; this is the case, for instance, in coupled dark energy models where the coupling occurs only with the dark matter.

${ }^{7}$ We do not further investigate the Symmetron, K-mouflage or Vainshtein models in this paper.
} 
These Dilaton models are scalar-tensor theories, where the action defining the system takes the general form

$$
\begin{aligned}
S= & \int \mathrm{d}^{4} x \sqrt{-g}\left[\frac{M_{\mathrm{Pl}}^{2}}{2} R-\frac{1}{2}(\nabla \varphi)^{2}-V(\varphi)-\Lambda_{0}^{4}\right] \\
& +\int \mathrm{d}^{4} x \sqrt{-\tilde{g}} \tilde{\mathcal{L}}_{m}\left(\psi_{m}^{(i)}, \tilde{g}_{\mu \nu}\right),
\end{aligned}
$$

where $M_{\mathrm{Pl}}=\left(8 \pi \mathcal{G}_{\mathrm{N}}\right)^{-1 / 2}$ is the reduced Planck mass (in natural units), $\Lambda_{0}^{4}$ is the cosmological constant term today, $g$ is the determinant of the Einstein-frame metric tensor $g_{\mu \nu}$ and $\tilde{g}$ is the determinant of the Jordan-frame metric tensor $\tilde{g}_{\mu \nu}$. The two metrics are connected via a conformal rescaling

$\tilde{g}_{\mu \nu}=A^{2}(\varphi) g_{\mu \nu}$.

The various matter fields $\psi_{m}^{(i)}$ are governed by the Jordan-frame Lagrangian density $\tilde{\mathcal{L}}_{m}$ and the scalar field $\varphi$ by the Einstein-frame Lagrangian density $\mathcal{L}_{\varphi}=-1 / 2(\nabla \varphi)^{2}-V(\varphi)$, where $V(\varphi)$ is the potential of the scalar field. ${ }^{8}$ There is no explicit coupling between matter and the scalar fields, and the fifth force on matter particles due to $\varphi$ arises from the conformal transformation given by equation (2) (more precisely, through gradients of $A$ ).

In the original Dilaton model, the potential $V(\varphi)$ and the coupling 9 $A(\varphi)$ with the metric have the following functional forms:

$V(\varphi)=V_{*} \exp \left(-\frac{\varphi}{M_{\mathrm{Pl}}}\right)$,

$A(\varphi)=1+\frac{1}{2} \frac{A_{2}}{M_{\mathrm{Pl}}^{2}} \varphi^{2}$,

where $\left\{V_{*}, A_{2}\right\}$ are the two free parameters. In regions where $\varphi \approx$ 0 , the coupling to matter is negligible, and gravity converges to GR. However, the field nevertheless mediates a long-range gravitational force that has an effect elsewhere, i.e. in less dense environments. This model can be generalized to a greater class of Dilaton models, by keeping the coupling function as in equation (4) but considering more general potentials. Then, instead of specifying the model by its potential $V(\varphi)$, it is recast in the tomographic parametrization $\{\beta(a), m(a)\}$ in terms of the cosmological scale factor $a(t)$, where the coupling $\beta(a)$ and the scalar field mass $m(a)$ are defined as (Brax et al. 2012c; Brax \& Valageas 2013):

$\beta(a) \equiv \beta[\bar{\varphi}(a)]=M_{\mathrm{Pl}} \frac{\mathrm{d} \ln A}{\mathrm{~d} \varphi}(\bar{\varphi})$,

$m^{2}(a) \equiv m^{2}[\bar{\varphi}(a), \bar{\rho}(a)]=\frac{1}{c^{2}}\left[\frac{\mathrm{d}^{2} V}{\mathrm{~d} \varphi^{2}}(\bar{\varphi})+\bar{\rho} \frac{\mathrm{d}^{2} A}{\mathrm{~d} \varphi^{2}}(\bar{\varphi})\right]$.

Hereafter, we denote with an overbar unperturbed cosmological background quantities, and with a subscript ' 0 ' quantities evaluated today. For instance, $\bar{\rho}(a)=3 \Omega_{\mathrm{m} 0} H_{0}^{2} M_{\mathrm{Pl}}^{2} / a^{3}$ is the background matter density, $\bar{\varphi}$ is the mean value of the field, $H_{0}$ is the current value of the Hubbles parameter, and $\Omega_{\mathrm{m} 0}$ is the current matter density.

\footnotetext{
${ }^{8}$ In equation (1), we explicitly added the cosmological constant term $\Lambda_{0}^{4}$, so that the minimum of $V(\varphi)$ is zero and is reached for $\varphi \rightarrow \infty$. Alternatively, this term could also be interpreted as the non-zero minimum of the scalar field potential.

${ }^{9}$ This coupling is often defined as $A(\varphi)=1+\frac{1}{2} \frac{A_{2}}{M_{\mathrm{Pl}}^{2}}\left(\varphi-\varphi_{*}\right)^{2}$, where $\varphi_{*}$ is some free parameter of the model. We opted to absorb $\varphi \star$ into $\varphi$ in equation (4), a choice that has no physical impact anyway.
}

Also, $c$ is the speed of light in vacuum. In this paper, we consider the simple forms in $m$ and $\beta$ :

$m(a)=m_{0} a^{-r}, \quad \beta(a)=\beta_{0} \exp \left[-s \frac{a^{2 r-3}-1}{3-2 r}\right]$,

with

$s=\frac{9 A_{2} \Omega_{\mathrm{m} 0} H_{0}^{2}}{c^{2} m_{0}^{2}}$.

Since the growth function involves only $m(a)$ and $\beta$, this allows for an efficient parametrization of both the background and the fluctuations, without having to model the potential nor the coupling function. In this framework, the Yukawa potential given by equation (3) corresponds to $r=3 / 2$. The values of the free parameters $\left\{m_{0}, r, \beta_{0}, s\right\}$ that enter equation (7) are displayed in Table 1. The models $\{\mathrm{A}, \mathrm{B}, \mathrm{C}, \mathrm{D}\}$ were chosen such as to correspond to those studied in Brax \& Valageas (2013) and Brax et al. (2012a), where detailed comparisons between numerical and analytical calculations are presented. More specifically, the models $\{\mathrm{A}, \mathrm{B}, \mathrm{C}\}$ probe the dependence on $\left\{s, \beta_{0}, r\right\}$ respectively, all other parameters being fixed, while models $\mathrm{D}$ probe the dependence on $m_{0}$ at fixed $A_{2}$. We added the models $E$ that probe the dependence on the parameter $m_{0}$ at fixed $\left\{s, \beta_{0}, r\right\}$. These models probe deviations from the $\Lambda \mathrm{CDM}$ cosmology of less than 20 per cent, in terms of the matter power spectrum. Let us now explore the detailed mechanism through which the power spectrum of matter fluctuations is affected by this theory.

The increase of the gravitational interaction due to the presence of the scalar field for all models described by the $\{m(a), \beta(a)\}$ parametrization results from the role played by the effective Newton constant $G_{\text {eff }}=\mathcal{G}_{\mathrm{N}}\left(1+\frac{2 \beta^{2}(a)}{1+m^{2}(a) a^{2} / k^{2}}\right)$ which is always larger than one. The effective Newton constant replaces Newton's constant $\mathcal{G}_{\mathrm{N}}$ in the equation driving the linear growth of the density contrast. In the non-linear regime, the scalar interaction is also attractive, leading to an increase of the clustering rate, albeit with a decreasing magnitude due to the Chameleon screening as one probes smaller scales. This generic feature applies to the Generalized Dilaton models (and to all viable $f(R)$ models, see the next section).

In these Dilaton models, the coupling function $A$ is always very close to unity, so that most Einstein-frame and Jordan-frame quantities (e.g. Hubble expansion rates or densities) are almost identical. Indeed, using $|\bar{A}-1| \ll 1$, we can see from equations (4) and (5) that $\bar{A} \simeq 1+\beta^{2} /\left(2 A_{2}\right)$. From equation (8), we also obtain $A_{2} \sim$ $\left(\mathrm{cm}_{0} / \mathrm{H}_{0}\right)^{2}$. Solar system tests of gravity ${ }^{10}$ such as that analysed in Chiba (2003) imply that $m_{0} \gtrsim 10^{3} H_{0} / c$, whence $A_{2} \gtrsim 10^{6}$ and

$|\bar{A}-1| \lesssim 10^{-6}$.

Therefore, the Jordan-frame and Einstein-frame scale factors and background matter densities, related by $\tilde{a}=\bar{A} a$ and $\tilde{\bar{\rho}}=\bar{A}^{-4} \bar{\rho}$, can be considered equal, as well as the cosmic times and Hubble expansion rates. In the rest of this section, we work in the Einstein frame, where the analysis of the gravitational dynamics is simpler.

In the Einstein frame, the Friedmann equation takes the usual form

$3 M_{\mathrm{Pl}}^{2} H^{2}=\bar{\rho}+\bar{\rho}_{\varphi}+\bar{\rho}_{\Lambda}$,

\footnotetext{
${ }^{10}$ Note that screening does not ensure zero deviation from GR, it only suppresses the deviation as compared with the linear regime prediction. Then, Solar system constraints need to be checked for each model. This provides constraints on the parameters that govern the efficiency of the screening mechanism.
} 
Table 1. Parameters describing the modified gravity theories considered in our study, mapped on the $\{\beta(a), m(a)\}$ surface, parametrized with $\left\{m_{0}, r, \beta_{0}, s\right\}$ following equation (7). The first five rows correspond to different realizations of the Generalized Dilaton theories. The last two rows show $f(R)$ theories with $n=1$ and 2, respectively, in which $m_{0}$ is given by equation (24), while $r \equiv 3(n+2) / 2, \beta_{0}=1 / \sqrt{6}$ and $s=0$.

\begin{tabular}{lccrr}
\hline Model & $m_{0}\left(h \mathrm{Mpc}^{-1}\right)$ & $r$ & $\beta_{0}$ & $s$ \\
\hline (A1, A2, A3) & $(0.334,0.334,0.334)$ & $(1.0,1.0,1.0)$ & $(0.5,0.5,0.5)$ & $(0.6,0.24,0.12)$ \\
(B1, B3, B4) & $(0.334,0.334,0.334)$ & $(1.0,1.0,1.0)$ & $(0.25,0.75,1.0)$ & $(0.24,0.24,0.24)$ \\
(C1, C3, C4) & $(0.334,0.334,0.334)$ & $(1.33,0.67,0.4)$ & $(0.5,0.5,0.5)$ & $(0.24,0.24,0.24)$ \\
(D1, D3, D4) & $(0.667,0.167,0.111)$ & $(1.0,1.0,1.0)$ & $(0.5,0.5,0.5)$ & $(0.06,0.96,2.16)$ \\
(E1, E3, E4) & $(0.667,0.167,0.111)$ & $(1.0,1.0,1.0)$ & $(0.5,0.5,0.5)$ & $(0.24,0.24,0.24)$ \\
$n=1, \log _{10}\left|f_{R_{0}}\right|=(-4,-5,-6)$ & $(0.042,0.132,0.417)$ & $(4.5,4.5,4.5)$ & $(0.408,0.408,0.408)$ & $(0,0,0)$ \\
$n=2, \log _{10}\left|f_{R_{0}}\right|=(-4,-5,-6)$ & $(0.034,0.108,0.340)$ & $(6.0,6.0,6.0)$ & $(0.408,0.408,0.408)$ & $(0,0,0)$ \\
\hline
\end{tabular}

where we explicitly separate contributions from the matter $(\bar{\rho})$ and scalar field $\left(\bar{\rho}_{\varphi}\right)$ components and from the cosmological constant $\bar{\rho}_{\Lambda}$. The background value of the scalar field potential is given by

$$
\frac{\mathrm{d} \bar{V}}{\mathrm{~d} \bar{\varphi}}+\frac{\beta}{M_{\mathrm{Pl}}} \bar{\rho}=0 \text {. }
$$

Combining with equation (6), and writing $m=m(a)$, this leads to

$$
\frac{\mathrm{d} \bar{\varphi}}{\mathrm{d} a}=\frac{3 \beta \bar{\rho}}{c^{2} M_{\mathrm{Pl}} a m^{2}}, \quad \frac{\mathrm{d} \bar{V}}{\mathrm{~d} a}=-\frac{3 \beta^{2} \bar{\rho}^{2}}{c^{2} M_{\mathrm{Pl}}^{2} a m^{2}},
$$

whence

$$
\frac{\dot{\bar{\varphi}}^{2}}{2 \bar{\rho}} \sim\left(\frac{H}{c m}\right)^{4} \sim 10^{-12}, \quad \frac{\bar{V}}{\bar{\rho}} \sim\left(\frac{H}{c m}\right)^{2} \sim 10^{-6} .
$$

Thus, the scalar field energy density is dominated by its potential term, which is negligible as compared with the matter density. Therefore, the Friedmann equation (10) is governed by the matter density and the cosmological constant and we recover the $\Lambda \mathrm{CDM}$ cosmological expansion, $3 M_{\mathrm{Pl}}^{2} H^{2}=\bar{\rho}+\bar{\rho}_{\Lambda}$, up to an accuracy of $10^{-6}$.

We now briefly consider the behaviour of metric and density fluctuations. We work within the quasi-static approximation, which can be shown to be the leading term in an expansion in $(H / \omega)^{2}$, where $\omega^{2}=k^{2} c^{2} / a^{2}+m^{2} c^{2}$ depends on both the comoving wavenumber $k$ and the inverse Compton wavelength $m$ of the scalar field. For $m \sim 0.1 h \mathrm{Mpc}^{-1}$ and $k=0$, we have $(H / \omega)^{2} \sim 10^{-5} ;(H / \omega)$ becomes even smaller as $k$ increases. Thus, for the models that we consider in this paper, the quasi-static approximation is valid for both the background and large-scale perturbations, which evolve on the Hubble time-scale, with a precision of $10^{-5}$ or better. ${ }^{11}$ In the quasi-static limit, then, the scalar field is given by the Klein-Gordon equation,

$$
\frac{c^{2}}{a^{2}} \nabla^{2} \varphi=\frac{\mathrm{d} V}{\mathrm{~d} \varphi}+\rho \frac{\mathrm{d} A}{\mathrm{~d} \varphi}
$$

and at linear order over the matter density and scalar field fluctuations we obtain

$$
\frac{\delta \varphi}{M_{\mathrm{Pl}}}=-\frac{\beta}{c^{2} M_{\mathrm{Pl}}^{2}} \frac{\delta \rho}{m^{2}+k^{2} / a^{2}},
$$

where $k$ is the comoving wavenumber. Using equation (11), this gives

$|\delta A| \sim \frac{|\delta \rho|}{\bar{\rho}}\left(\frac{H}{\mathrm{~cm}}\right)^{2} \frac{1}{1+k^{2} / a^{2} m^{2}} \lesssim 10^{-6}$,

\footnotetext{
${ }^{11}$ The quasi-static approximation breaks down if the scalar-field potential or coupling function show some singularity or sharp features (see Brax \& Valageas 2013 for a detailed analysis), but we do not consider such models here.
}

so that the perturbations of the conformal factor $A^{2}$ are negligible compared to unity. Also,

$$
\frac{\delta \rho_{\varphi}}{\delta \rho} \sim\left(\frac{H}{c m}\right)^{2} \frac{1}{1+k^{2} / a^{2} m^{2}} \lesssim 10^{-6},
$$

hence fluctuations of the scalar field energy density are negligible compared with the matter density fluctuations.

Therefore, the main source that drives modifications to structure growth is not a different background evolution, nor perturbations in the scalar field energy density, but really the action of the fifth force on the matter field. In the Newtonian gauge, the perturbed metric can be written as

$\mathrm{d} s^{2}=-(1+2 \Phi) \mathrm{d} t^{2}+a^{2}(t)(1-2 \Psi) \delta_{i j} \mathrm{~d} x^{i} \mathrm{~d} x^{j}$,

where $\Phi$ and $\Psi$ are the Einstein-frame metric gravitational potentials. Using equations (15) and (17), we can check that the impact of the scalar field fluctuations on the metric potentials are negligible, and we have within a $10^{-6}$ accuracy

$\Phi=\Psi=\Psi_{\mathrm{N}}$,

where $\Psi_{\mathrm{N}}$ is the Newtonian potential given by the Poisson equation,

$\frac{\nabla^{2}}{a^{2}} \Psi_{\mathrm{N}}=4 \pi \mathcal{G}_{\mathrm{N}} \delta \rho=\frac{3 \Omega_{\mathrm{m} 0} H_{0}^{2}}{2 a^{3}} \delta$.

In the above expression, $\delta=\delta \rho / \bar{\rho}$ is the matter density contrast. However, the dynamics of matter particles are modified by the scalar field, which gives rise to the fifth force given by $\boldsymbol{F}=-c^{2} \nabla \ln A$. That is, in the Euler equation we must add a fifth-force potential, $\Psi_{A}=c^{2} \ln A$, that is not negligible. When solving for structure growth given the parameters listed in Table 1, the new term can lead to $10-20$ per cent deviations in the matter density power spectrum.

\subsection{Gravity in $f(R)$ theories}

In models based on $f(R)$ gravity, the Einstein-Hilbert action is modified by promoting the Ricci scalar $R$ to a function of $R$ (Buchdahl 1970; Starobinsky 1980, 2007; Hu \& Sawicki 2007). The new action $S$ in $f(R)$ gravity theories can be written as

$S=\int \mathrm{d}^{4} x \sqrt{-g}\left[\frac{M_{\mathrm{Pl}}^{2}}{2}[R+f(R)]-\Lambda_{0}^{4}+\mathcal{L}_{m}\left(\psi_{m}^{(i)}\right)\right]$,

where we explicitly added the cosmological constant contribution. ${ }^{12}$ The $f(R)$ models are most easily described in the Jordan frame,

\footnotetext{
${ }^{12}$ The terms $R$ and $\Lambda_{0}^{4}$ are often included within the function $f(R)$. Written in the form of equation (21), $f(R)$ describes deviations from both GR and the $\Lambda \mathrm{CDM}$ cosmology.
} 
which is why, in this section, we denote with a tilde Einstein-frame quantities instead of Jordan-frame ones, ${ }^{13}$ contrary to the notation of Section 2.1. In the parametrization of Hu \& Sawicki (2007), the functional form $f(R)$ can be expressed in the high curvature limit as

$f(R)=-\frac{f_{R_{0}}}{n} \frac{R_{0}^{n+1}}{R^{n}}, \quad f_{R} \equiv \frac{\mathrm{d} f(R)}{\mathrm{d} R}=f_{R_{0}} \frac{R_{0}^{n+1}}{R^{n+1}}$.

The two independent parameters, $f_{R_{0}}$ and $n$, can be constrained by observations. In the above expression, $R_{0}$ is the present value of the Ricci scalar for the cosmological background. Note that this parametrization and that of Starobinsky (2007) both reproduce the same results in the large curvature regime.

The $f(R)$ theories of gravity also invoke the Chameleon mechanism to screen modifications of GR in dense environments such as in our Solar system. Specifically, this occurs by requiring that all extra terms vanish in high-curvature environment, such that $f\left(|R| \gg\left|R_{0}\right|\right) \rightarrow 0$. The background expansion otherwise follows the $\Lambda \mathrm{CDM}$ dynamics and the growth of structure is only affected on intermediate and quasi-linear scales.

There is an essential connection between the formulation of the $f(R)$ theory presented above and scalar-tensor theories of modified gravity. Upon the coordinate rescaling $\tilde{g}_{\mu \nu}=A^{-2}(\varphi) g_{\mu \nu}$ (recall that in this section $\tilde{g}_{\mu \nu}$ is the Einstein-frame metric) with $A(\varphi)=\exp \left[\beta \varphi / M_{\mathrm{Pl}}\right]$ and $\beta=1 / \sqrt{6}$, the $f(R)$ modifications to GR are recast as arising from contributions of an extra scalar field $\varphi$, subject to a potential $V(\varphi)$ given by

$V(\varphi)=\frac{M_{\mathrm{Pl}}^{2}}{2}\left(\frac{R f_{R}-f(R)}{\left(1+f_{R}\right)^{2}}\right), \quad f_{R}=\exp \left[-\frac{2 \beta \varphi}{M_{\mathrm{Pl}}}\right]-1$.

In that sense, $f(R)$ theories are equivalent to a scalar-tensor theory expressed in the Einstein frame (Chiba 2003; Nunez \& Solgnaik 2004). In this new formulation, the screening mechanism takes another form: the mass of the scalar field grows with matter density, and a Yukawa-like potential suppresses the fifth force in dense environments. This can be conveniently reformulated by saying that screening takes place wherever the scalar field is small compared to the ambient Newtonian potential.

It turns out that all Chameleon-like models such as $f(R)$ theories can again be parametrized by the value of the mass $m(a)$ and the coupling $\beta(a)$ of the scalar field, in terms of the scale factor $a$ and the associated background matter density $\bar{\rho}(a)$. With the specific functional form of $f(R)$ given by equation (22), we can directly relate $\left\{n, f_{R_{0}}\right\}$ to $\{\beta(a), m(a)\}$ via

$$
\begin{aligned}
m(a) & =m_{0}\left(\frac{4 \Omega_{\Lambda 0}+\Omega_{\mathrm{m} 0} a^{-3}}{4 \Omega_{\Lambda 0}+\Omega_{\mathrm{m} 0}}\right)^{(n+2) / 2}, \\
m_{0} & =\frac{H_{0}}{c} \sqrt{\frac{\Omega_{\mathrm{m} 0}+4 \Omega_{\Lambda 0}}{(n+1)\left|f_{R_{0}}\right|}}, \quad \beta(a)=\frac{1}{\sqrt{6}} .
\end{aligned}
$$

In this paper, we consider values of $n=\{1,2\}$ and $\left|f_{R_{0}}\right|=$ $\left\{10^{-4}, 10^{-5}, 10^{-6}\right\}$. The larger value of $\left|f_{R_{0}}\right|$ is currently ruled out

\footnotetext{
${ }^{13}$ We have decided to define the Generalized Dilaton models in the Einstein frame and the $f(R)$ models in the Jordan frame, mainly for simplicity in the notation. The $f(R)$ models can be recast in the Einstein frame (with equation 23 ) if necessary. Note that in the Einstein frame, there is only one metric potential, as $\Phi=\Psi=\Psi_{\mathrm{N}}$ in equation (19), whereas in the Jordan frame the two metric potentials are distinct, $\Phi \neq \Psi$, see equation (28). In the end however, the weak lensing potential (equation 32) is uniquely defined and coincides in both frames. As a result, we freely use either frames when convenient.
}

by other independent probes, so this serves as a consistency test. The numerical values for $\{\beta(a), m(a)\}$ corresponding to these three models are listed in Table 1.

As for the Dilaton models described in Section 2.1, the $f(R)$ models that we consider in this paper follow very closely the $\Lambda \mathrm{CDM}$ cosmology at the background level, mainly because $\left|f_{R_{0}}\right| \ll 1$. Indeed, from the action (equation 21) one obtains the Friedmann equation as (Tsujikawa 2007)

$3 M_{\mathrm{Pl}}^{2}\left[H^{2}-\bar{f}_{R}\left(H^{2}+\dot{H}\right)+\bar{f} / 6+\bar{f}_{R R} H \dot{\bar{R}}\right]=\bar{\rho}+\bar{\rho}_{\Lambda}$,

where the dot denotes the derivative with respect to cosmic time $t$ and $f_{R R}=\mathrm{d}^{2} f / \mathrm{d} R^{2}$. In the background, we have $\bar{R}=12 H^{2}+6 \dot{H}$ and we can check that all extra terms in the brackets in equation (25) are of the order of $\left|f_{R_{0}}\right| H^{2}$, so that we recover the $\Lambda \mathrm{CDM}$ expansion, $3 M_{\mathrm{Pl}}^{2} H^{2}=\bar{\rho}+\bar{\rho}_{\Lambda}$, up to an accuracy of $10^{-4}$ for $\left|f_{R_{0}}\right| \lesssim 10^{-4}$. Moreover, the conformal factor $A(\varphi)$ is given by $A=\left(1+f_{R}\right)^{-1 / 2}$, so that $|\bar{A}-1| \lesssim 10^{-4}$ and the background quantities associated with the Einstein and Jordan frames can be considered equal (and equal to the $\Lambda \mathrm{CDM}$ reference) up to an accuracy of $10^{-4}$.

Considering the metric and density perturbations, we can again write the Newtonian gauge metric as in equation (18) (but this is now the Jordan-frame metric). Then, in the small-scale sub-horizon limit $k / a \gg H / c$, the modified Einstein equations lead to (Tsujikawa $\&$ Tatekawa 2008)

$\frac{\nabla^{2}}{a^{2}} \Phi=-\frac{c^{2} \nabla^{2}}{2 a^{2}} \delta f_{R}+4 \pi \mathcal{G}_{\mathrm{N}} \delta \rho$,

$\frac{\nabla^{2}}{a^{2}} \Psi=\frac{c^{2} \nabla^{2}}{2 a^{2}} \delta f_{R}+4 \pi \mathcal{G}_{\mathrm{N}} \delta \rho$,

where $\delta f_{R}=f_{R}-\bar{f}_{F}, \delta \rho=\rho-\bar{\rho}$, and using the approximation $\left|f_{R}\right| \ll 1$. Therefore, in terms of the Newtonian gravitational potential $\Psi_{\mathrm{N}}$ defined as in GR by equation (20), we have

$\Phi=\Psi_{\mathrm{N}}-\frac{c^{2}}{2} \delta f_{R}, \quad \Psi=\Psi_{\mathrm{N}}+\frac{c^{2}}{2} \delta f_{R}$.

Thus, because we work in the Jordan frame (in contrast with the Dilaton case presented in Section 2.1), the modification of gravity directly appears through the metric potentials. The fluctuations of the new degree of freedom $\delta f_{R}$ are given by

$\frac{3 c^{2} \nabla^{2}}{a^{2}} \delta f_{R}=\delta R-8 \pi \mathcal{G}_{\mathrm{N}} \delta \rho$.

Finally, the dynamics of the matter particles is given by the geodesic equation, where the Newtonian potential that appears in GR is replaced by the potential $\Phi$ given in equation (28).

\section{WEAK LENSING}

\subsection{Theory}

\subsubsection{Weak lensing convergence power spectrum}

In all the cosmologies considered in this paper, we work in the Newtonian gauge with the perturbed metric given by equation (18), where $\Phi$ and $\Psi$ are the metric gravitational potentials. ${ }^{14}$ In practice,

\footnotetext{
${ }^{14}$ In the Dilaton models, this is understood as the Einstein-frame metric while in the $f(R)$ models this is the Jordan-frame metric, following the approach described in Section 2. In any case, we can work in either frame as the observational results do not depend on this computational choice.
} 
we measure the statistical properties of weak lensing distortions by summing over many galaxy images. This means that the measured signal is an integral over selected sources with a broad redshift distribution $n\left(z_{\mathrm{s}}\right) \mathrm{d} z_{\mathrm{s}}$ (mapped to $n(\chi) d \chi$ in terms of the radial distance, given the Jacobian $\mathrm{d} \chi / \mathrm{d} z$ ) that we normalize to unity. Thus, introducing the kernel $g(\chi)$ that defines the radial depth of the survey:

$g(\chi)=\int_{\chi}^{\infty} \mathrm{d} \chi_{s} n\left(\chi_{s}\right) \frac{\chi_{s}-\chi}{\chi_{s}}$

the integrated convergence field at a position $\boldsymbol{\theta}$ on the sky reads as

$\kappa(\boldsymbol{\theta})=\int_{0}^{\infty} \mathrm{d} \chi \frac{\chi}{c^{2}} g(\chi) \nabla^{2} \Phi_{\mathrm{wl}}(\chi, \chi \boldsymbol{\theta})$.

We assumed a flat background universe in the above equation, and introduced the weak lensing potential, defined by

$\Phi_{\mathrm{wl}}=\frac{\Phi+\Psi}{2}$,

which is convenient when computing weak lensing modifications to GR. Solving equation (31) in multipole space and taking the ensemble average of the squared complex norm, we obtain the convergence power spectrum

$C_{\ell}^{\kappa}=\int_{0}^{\infty} \mathrm{d} \chi \frac{g(\chi)^{2}}{c^{4}} \frac{\ell^{4}}{\chi^{4}} P_{\Phi_{\mathrm{wl}}}(\ell / \chi ; z)$

as an integral over the weak lensing power spectrum $P_{\Phi_{\mathrm{wl}}}(k ; z)$. Note that the above also assumes both Limber and Born approximations. From this, we also derive predictions for the cosmic shear two-point correlation functions $\xi_{ \pm}(\theta)$, computed as

$\xi_{ \pm}(\theta)=\frac{1}{2 \pi} \int C_{\ell}^{\kappa} J_{0 / 4}(\ell \theta) \ell \mathrm{d} \ell$,

where $J_{0 / 4}(x)$ are Bessel functions of the first kind.

\section{$3.1 .2 C_{\ell}^{\kappa}$ in $G R$}

In the $\Lambda \mathrm{CDM}$ cosmology + GR case, we can exactly express the weak lensing convergence power spectrum (33) in terms of the total matter power spectrum $P(k)$ via Poisson equation. Indeed, we can safely neglect the anisotropic stress, and GR gives

$\Phi_{\mathrm{wl}}=\Phi=\Psi=\Psi_{\mathrm{N}}$,

where $\Psi_{\mathrm{N}}$ is the Newtonian potential given by Poisson equation (equation 20). Therefore, we recover

$P_{\Phi_{\mathrm{wl}}}(k ; z)=\left(\frac{3 \Omega_{\mathrm{m} 0} H_{0}^{2}}{2 a k^{2}}\right)^{2} P(k ; z)$

and the convergence power spectrum (33) becomes

$C_{\ell}^{\kappa}=\int_{0}^{\infty} \mathrm{d} \chi W(\chi)^{2} P(\ell / \chi ; z)$,

with

$W(\chi)=\frac{3 \Omega_{\mathrm{m} 0} H_{0}^{2}}{2 c^{2}} g(\chi)(1+z)$.

\subsection{3 $C_{\ell}^{\kappa}$ in theories of modified gravity}

For the Dilaton models, we have seen in equation (19) and in Section 2.1 that the two Einstein-frame metric potentials are equal to the Newtonian potential up to order $10^{-6}$ accuracy, and that background cosmological quantities such as the Hubble expansion rate and the radial comoving distances are equal to those of the $\Lambda \mathrm{CDM}$ reference within that same accuracy. This means that equations (35) and (36) apply as in GR, and that $C_{\ell}^{\kappa}$ is again given by equations (37) and (38). Therefore, in terms of this weak lensing statistics, the modification of gravity and the departures from the $\Lambda \mathrm{CDM}+\mathrm{GR}$ results only appear through the modified matter density power spectra $P(k ; z)$, which we describe in Section 3.2.3.

In the case of $f(R)$ models, we have seen in equation (28) that the two Jordan-frame potentials are different from the Newtonian potentials, receiving contributions from terms linear in $\delta f_{R}$. However, these two extra terms exactly cancel in the weak lensing potential (equation 32) such that $\Phi_{\mathrm{wl}}=\Psi_{\mathrm{N}}$. Therefore, we recover equations (36) - (38) in $f(R)$ models too. Moreover, we have seen that both the Jordan-frame and Einstein-frame background quantities are equal to the reference $\Lambda \mathrm{CDM}$ background quantities up to an accuracy of $10^{-4}$ for $\left|f_{R_{0}}\right| \lesssim 10^{-4}$. This means that weak lensing statistics can again be computed in the reference background cosmology, so long as the modified matter density power spectrum is used.

\subsection{Non-linear matter power spectrum}

The choice of non-linear power spectrum to insert in equation (37) depends on the cosmology under investigation. In this paper, we are interested in constraining modified gravity models, but with respect to a $\Lambda \mathrm{CDM}$ baseline, these are strongly degenerated with universes that include baryon feedbacks and/or massive neutrinos. In the context of cosmic shear, these phenomena are therefore intrinsically connected and must be jointly analysed. We detail in this section how we combine all these effects in the construction of our theoretical predictions.

\subsubsection{Dark matter only}

The first choice we make concerns the dark matter model $P^{\mathrm{DM}}(k)$, which is a delicate issue that has been thoroughly investigated in HWVH in a very similar context. Following this work, we choose the dark matter only (DM-ONLY) model that best reproduces the results from a number of $\Lambda \mathrm{CDM} N$-body simulations, then implement the combined effect of modified gravity, baryon feedback and massive neutrinos relative to this DM-ONLY baseline. Our DM-ONLY prediction is a hybrid model that combines the Extended Cosmic Emulator (Heitmann et al. 2014) with the recalibrated HALOFIT code by Takahashi et al. (2012). Its convergence properties have been well examined in HWVH, and it was shown to have the best agreement with independent high-resolution simulation suites, compared with other models. In addition, HWVH examined the scatter across multiple models, and estimated the theoretical uncertainty on the global DM-ONLY prediction for $\xi_{ \pm}$. In this paper, we also incorporate this model uncertainty in the analysis pipeline, at the level of the $\chi^{2}$ calculation (see Section 4.1).

\subsubsection{Neutrino and baryon feedback}

Following HWVH, we model the impact of massive neutrinos and baryon feedback on the matter power spectrum as separate effects that can be expressed with multiplicative feedback terms, namely,

$P^{\mathrm{DM}+v+b(\mathrm{~m})}(k, z)=P^{\mathrm{DM}}(k, z) \times b_{M_{\nu}}^{2}(k, z) \times b_{\mathrm{m}}^{2}(k, z)$.

The underlying assumption is that both biases are independent, which is reasonable since baryons were found to have a 1 per cent 
effect on the neutrinos bias for $k<8 \mathrm{~h} \mathrm{Mpc}^{-1}$ (Bird, Viel \& Haehnelt 2012).

We compute the neutrino feedback bias term $b_{M_{v}}^{2}$ with the CAMB cosmological code (Lewis, Challinor \& Lasenby 2000), which is reported to be accurate to better than 10 per cent at $k=10 \mathrm{~h} \mathrm{Mpc}^{-1}$ (Bird et al. 2012). We assume one massive neutrino flavour, and fix the cosmology at high redshift - i.e. we keep the primordial amplitude $A_{\mathrm{s}}$ fixed but let $\sigma_{8}$ vary. We justify this choice from the fact that the former quantity is measured very accurately by CMB observations, whereas our estimation of the latter quantity is much less accurate due to galactic and cluster bias. We construct the neutrino bias as

$b_{M_{\nu}}^{2}(k, z) \equiv \frac{P_{\mathrm{CAMB}}^{\mathrm{DM}+M_{\nu}}(k, z)}{P_{\mathrm{CAMB}}^{\mathrm{DM}}(k, z)}$,

where the $M_{v}(=0.0,0.2,0.4$ or $0.6 \mathrm{eV})$ superscript specifies the total neutrino mass considered, and the subscript 'CAMB' specifies that both quantities are measured from this cosmological numerical code.

The baryonic feedback bias is estimated from two hydrodynamical simulations ran in the context of the OverWhelmingly Large (OWL) Simulation Project (Schaye et al. 2010). The DM-ONLY run is a purely collisionless $N$-body calculation and acts as the baseline for this baryon feedback measurement only. The active galactic nucleus (AGN) simulation run contains gas dynamics with physical prescriptions for cooling, heating, star formation and evolution, chemical enrichment, supernovae feedback andAGN feedback (see van Daalen et al. 2011 for details about these simulations). Following van Daalen et al. (2011) and Semboloni et al. (2011), we measure the baryonic feedback bias by taking the ratio between the AGN and the DM-ONLY models ${ }^{15}$ :

$b_{\mathrm{m}}^{2}(k, z) \equiv \frac{P_{\mathrm{OWL}}^{\mathrm{DM}+b(\mathrm{~m})}(k, z)}{P_{\mathrm{OWL}}^{\mathrm{DM}}(k, z)}$,

where the index $b(\mathrm{~m})$ refers to either DM-ONLY or AGN, and the subscript 'OWL' specifies that these quantities were measured specifically from the OWL simulation suite.

Fig. 1 shows the impact of different combinations of baryons and massive neutrinos on the matter power spectrum. Figs 2 and 3 show the equivalent effects on the weak lensing power spectrum $C_{\ell}^{\kappa}$ and on the shear two-point correlation function $\xi_{ \pm}(\theta)$, respectively. We can see from the three figures that all models converge to DM-ONLY at large scales (low $k$, low $\ell$ and high $\theta$ ), and that the combined effect can suppress more than 50 per cent of the power, depending on the models and neutrino mass. Also, it becomes clear that surveys probing small patches (restricted to $\ell>500$ for example) would have difficulties to distinguish between the two feedback processes. This degeneracy can only be broken with the inclusion of lower $\ell$ multipoles, where baryon feedback is minimal but massive neutrinos still leave a signature (Natarajan et al. 2014).

\subsubsection{Combined feedback with modified gravity}

The evolution of perturbations in the context of large-scale structures has been carefully studied in $f(R)$ and scalar-tensor theories' gravity (Koivisto 2006; Zhang 2006; Bean et al. 2007; Hu \& Sawicki 2007; Song et al. 2007a,b; Carloni, Dunsby \& Troisi 2008; Pogosian \& Silvestri 2008; Koyama, Taruya \& Hiramatsu 2009;

\footnotetext{
15 The power spectrum measurements from the OWL simulation suite are
} publicly available at http://vd11.strw.leidenuniv.nl

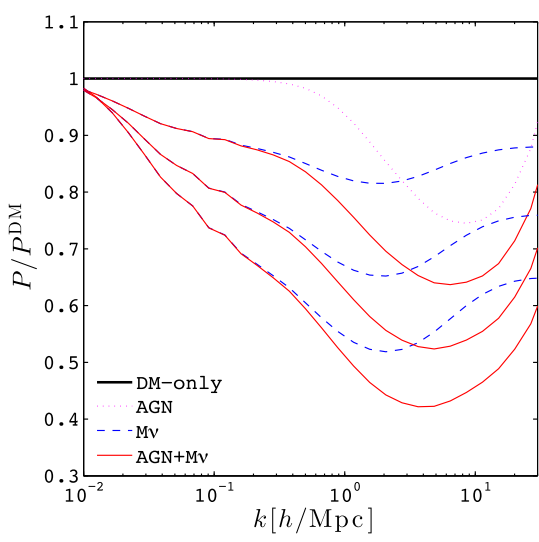

Figure 1. Combined effect from baryon feedback and massive neutrinos on the matter power spectrum $P(k)$, evaluated at $z=1$. Results are shown with respect to the DM-ONLY non-linear predictions (thick solid line). From top to bottom, the (blue) dashed lines represent the effect of massive neutrinos with $M_{v}=0.2,0.4$ and $0.6 \mathrm{eV}$, respectively. The combinations of massive neutrinos with baryon feedback are shown with the thin (red) solid lines.

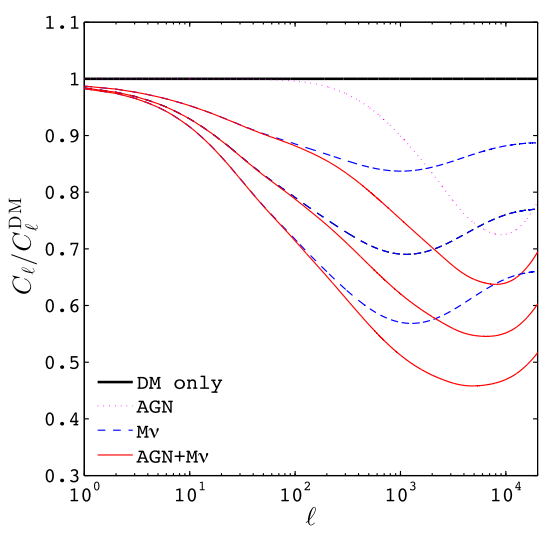

Figure 2. Combined effect from baryon feedback and massive neutrinos on the weak lensing power spectrum, assuming the source redshift distribution given by equation (45) and the baseline WMAP9 cosmology. As for Fig. 1, results of different combinations are shown with respect to the DM-ONLY non-linear predictions (thick solid line), and the sum of neutrino masses shown are, from top to bottom, $M_{v}=0.2,0.4$ and $0.6 \mathrm{eV}$.
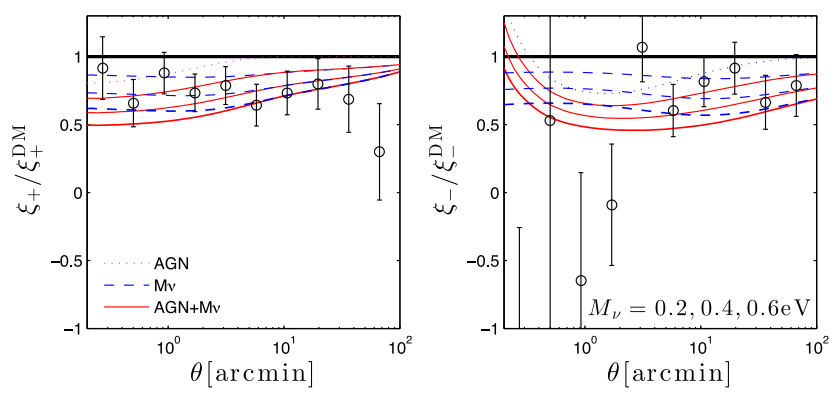

Figure 3. Left: combined effect from baryon feedback and massive neutrinos on the weak lensing two-point correlation function $\xi_{+}$. The open symbols represent our measurements from CFHTLenS data, shown with $1 \sigma$ error bars. Right: same as the left-hand panel, but for the $\xi$ - estimator. We used the same $y$-axis range for both panels to emphasize on the differences across the models, hence the leftmost point falls outside the frame, at $\xi_{-} / \xi_{-}^{\mathrm{DM}}=-3.8$. 
Motohashi, Starobinsky \& Yokoyama 2009; Brax et al. 2011; Li \& Hu 2011; Li, Zhao \& Koyama 2012; Linares \& Mota 2013; Brax \& Valageas 2013; Taddei, Catena \& Pietroni 2014). In this paper, we focus on the matter density power spectrum $P(k ; z)$, or more precisely, on the weak lensing convergence power spectrum $C_{\ell}^{\kappa}$, which can be computed from $P(k ; z)$ through the modified Poisson equations that relate the metric gravitational potentials to the matter density fluctuations.

Therefore, before computing weak lensing statistics, we first need to describe gravitational clustering and the $3 \mathrm{D}$ matter density power spectrum for all cosmological scenarios that we investigate. We use the approach first developed in Valageas, Nishimichi \& Taruya (2013) for the $\Lambda$ CDM cosmology, generalized afterwards to various modified-gravity scenarios in Brax \& Valageas (2013, 2014b). This is an analytical approach that combines perturbation theory up to one-loop order (i.e. up to order $P_{\mathrm{L}}^{2}$, where $P_{\mathrm{L}}$ is the linear matter density power spectrum) with a phenomenological halo model. Namely, we are splitting the matter power spectrum as

$P(k)=P_{2 \mathrm{H}}(k)+P_{1 \mathrm{H}}(k)$,

where $P_{2 \mathrm{H}}(k)$ is the 'two-halo' term associated with pairs of particles that are enclosed in two different haloes, whereas $P_{1 \mathrm{H}}(k)$ is the 'one-halo' term associated with pairs enclosed in the same halo. This construction allows us to obtain predictions for the non-linear matter power spectrum covering the linear, quasi-linear and highly non-linear scales. We refer the reader to the work cited above for complete details and validations of equation (42), but nevertheless provide an overview of the method in Appendix A for quick reference. We note that other prescriptions exist for modelling $P(k)$ in modified gravity scenarios, i.e. Zhao (2014) for the $f(R)$ model. However the modelling we adopt here applies also to $f(R)$ with $n$ $\neq 1$ gravity, to Dilaton gravity, and in fact to any modified gravity model expressed in the tomographic parametrization, which makes it general and accurate at the same time.

In analogy with equations (40) and (41), we define the modified gravity bias:

$b_{\mathrm{MG}(\alpha)}^{2}(k, z) \equiv \frac{P_{\mathrm{VNT}}^{\mathrm{MG}(\alpha)}(k, z)}{P_{\mathrm{VNT}}^{\mathrm{DM}}(k, z)}$,

where $\operatorname{MG}(\alpha)$ refers to the gravity model, with $\alpha=0$ corresponding to $\mathrm{GR}, \alpha=[1,2,3, \ldots, 15]$ specifying dilation models [A1, A2, A3, . . E4], $\alpha=[16,17,18]$ specifying $f(R)$ models with $n=1$ and $\left|f_{R_{0}}\right|=10^{-4}, 10^{-5}, 10^{-6}$, and finally $\alpha=[19,20,21]$ the $f(R)$ models with $n=2$ and the same $\left|f_{R_{0}}\right|$ values. The subscript 'VNT' indicates quantities that are computed in the framework of Valageas et al. (2013), i.e. with equation (42).

Bringing all the pieces together, we construct the matter power spectrum for any combination of baryon feedback, neutrino mass and modified gravity by multiplying the DM-ONLY model by the corresponding biases:

$P^{\mathrm{DM}+v+b(\mathrm{~m})+\mathrm{MG}}=P^{\mathrm{DM}} \times b_{M_{v}}^{2} \times b_{\mathrm{m}}^{2} \times b_{\mathrm{MG}(\alpha)}^{2}$.

We have removed the dependences on scale and redshift for each of these terms to clarify the notation. This modelling assumes that the effect of modified gravity on the baryon and neutrino feedbacks can be neglected, allowing for the convenient factorization presented in equation (44). This seems to be a valid approximation for some models, as it was shown in Hammami et al. (2015) that the modified gravity bias measured in DM-ONLY matched to better than 5 per cent the same measurement done in full hydrodynamical simulations, for $f(R)$ models with $n=1$ and $\left|f_{R_{0}}\right| \in\left[10^{-4}\right.$ to $\left.10^{-6}\right]$. However, the same group also observed larger deviations in many
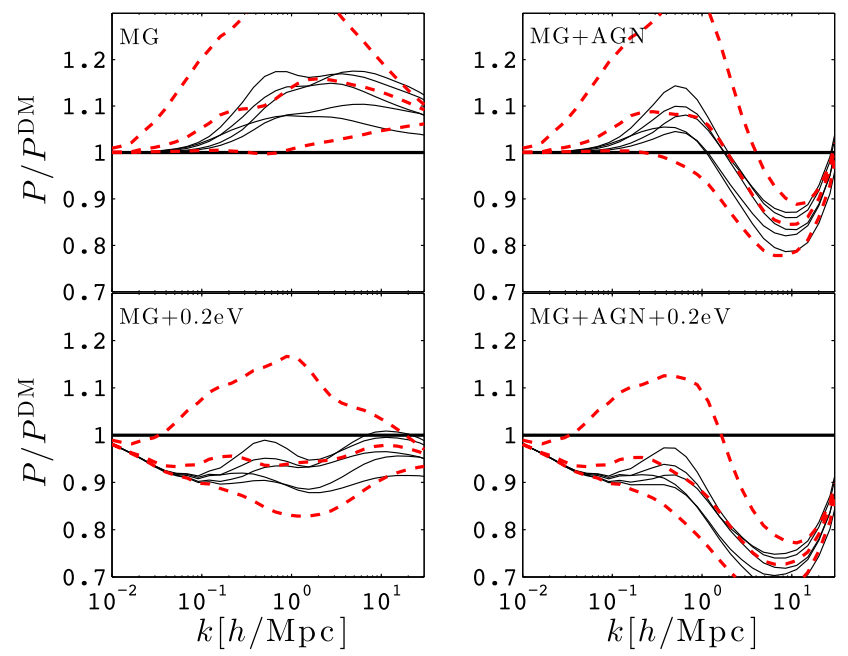

Figure 4. Combined effect from baryon feedback and massive neutrinos on the matter power spectrum $P(k)$ assuming different modified gravity models, again evaluated at $z=1$. Results are shown with respect to the DM-ONLY non-linear predictions (thick horizontal solid line). From top to bottom at $k$ $=0.2 \mathrm{~h} \mathrm{Mpc}^{-1}$, the solid lines represent Dilaton models B4, A3, E3, D1 and $\mathrm{C} 1$, respectively. The thick red dashed lines correspond to $f(R)$ gravity with $n=1$. Top to bottom are for $\left|f_{R_{0}}\right|=10^{-4}, 10^{-5}$ and $10^{-6}$, respectively. We do not show the $n=2$ results to avoid overcrowding the figure, but they are qualitatively similar in shape to the $n=1$ case, albeit with a smaller departure from $\Lambda \mathrm{CDM}$. Different panels show different combinations of massive neutrinos and baryon feedback on these same models, all computed with equation (44).

symmetron models, up to 20 per cent by $k=10 \mathrm{~h} \mathrm{Mpc}^{-1}$ in some cases. This places a limit on the accuracy of equation (44), and calls for more hydrodynamical simulation runs where $b_{\mathrm{m}}$ and $b_{\mathrm{MG}(\alpha)}$ are merged into one term, $b_{\mathrm{m}, \mathrm{MG}(\alpha)}$, measured for each combination of $\{\alpha, m\}$. This is unfortunately not available at the moment, hence equation (44) is currently our best shot at this joint measurement. On the neutrino sector, results by Baldi et al. (2014) are further encouraging: they looked at joint simulations of modified gravity and massive neutrinos and came to the conclusion that one could consider the effect of each almost independently, supporting the validity of equation (44).

For each combination, we compute predictions for the weak lensing quantity with equations (37) and (34). We report our results on $P(k)$ and $C_{\ell}^{\kappa}$ in Figs 4 and 5, respectively. Whereas modified gravity is generally boosting the clustering compared to a $\Lambda \mathrm{CDM}$ universe, the inclusion of massive neutrinos and/or baryonic feedback is working in the opposite direction. It becomes clear that a precise distinction between these three feedback contributions poses a challenge to clustering and weak lensing experiments.

\subsection{Data}

Our measurement of the shear correlation functions $\xi_{ \pm}$is based on the public release of the CFHTLenS. ${ }^{16}$ The CFHTLenS covers a total area of $154 \mathrm{deg}^{2}$, which is reduced to $128 \mathrm{deg}^{2}$ after masking bright stars, foreground moving objects and faulty CCD rows. Full details about the data reduction pipeline are provided in Erben et al. (2013). Source redshifts are obtained from the five bands $u^{\prime}$ griz photometric observations (Hildebrandt et al. 2012) and were

${ }^{16}$ CFHTLenS: www.cfhtlens.org 

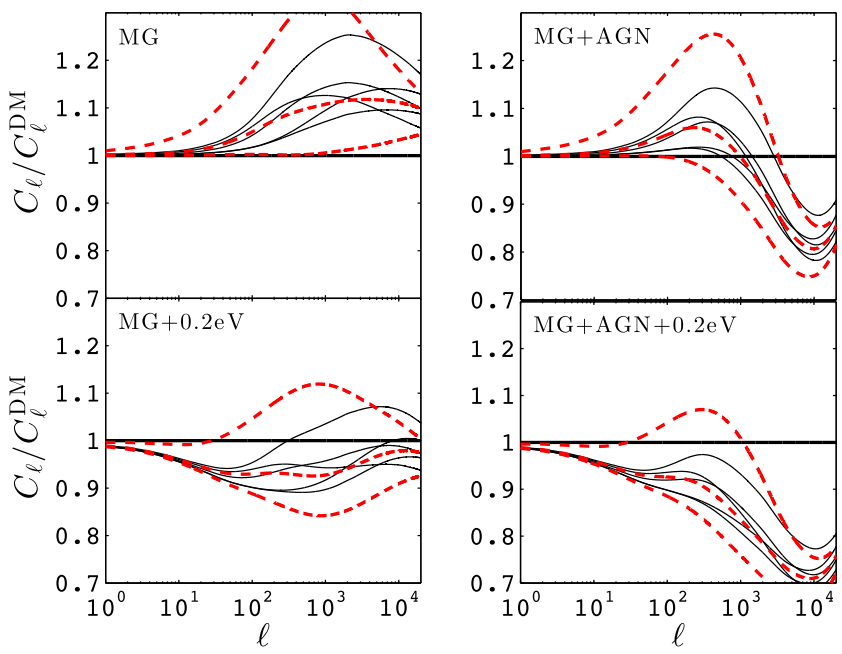

Figure 5. Same as Fig. 4, but for the weak lensing power spectrum.

carefully tested in Benjamin et al. (2013); shape measurements are performed on the $r$-band images with the LENSFIT Bayesian code described in Miller et al. (2013). A detailed assessment of the residual systematics is provided in Heymans et al. (2012), and we refer the reader to these references for more information on the CFHTLenS data.

As described in Heymans et al. (2012), the public shear data must be recalibrated with additive and multiplicative factors, commonly referred to as the $c$ and $m$ corrections. In contrast with this reference, we use a different $c$ correction, as detailed in HWVH, which is less model dependent. Although the overall change on the correction is marginal, the number of CFHTLenS pointings that are flagged as bad is reduced by almost a half.

Following the recommendations of Heymans et al. (2012) and Benjamin et al. (2013), we minimize the systematic contamination from badly reconstructed photometric redshifts by applying the selection cut $0.4<z_{\text {phot }}<1.3$. We construct the redshift distribution $n(z)$ for the selected galaxies from the LENSFIT-weighted stacked probability distribution functions of the galaxy sample. As shown in $\mathrm{HWMH}$, the distribution is well described by the following analytical expression:

$$
\begin{aligned}
n(z)= & N_{0} \mathrm{e}^{-\left(z-z_{0}\right)^{2} / \sigma_{0}^{2}}+N_{1} \mathrm{e}^{-\left(z-z_{1}\right)^{2} / \sigma_{1}^{2}} \\
& +\frac{N_{2} \mathrm{e}^{-\left(z-z_{2}\right)^{2} / \sigma_{2}^{2}}}{1.0+\mathrm{e}^{-10.0(z-0.6)}},
\end{aligned}
$$

where $\left(N_{0}, z_{0}, \sigma_{0}, N_{1}, z_{1}, \sigma_{1}, N_{2}, z_{2}, \sigma_{2}\right)=(0.14438,0.760574$, $0.14594,0.514894,0.498379,0.15608,1.74435,0.445019$, 0.684098 ). There is a 0.4 per cent difference in the mean redshift between the fit and the distribution, which yields a small error well below the other sources of error in our analysis. We therefore neglected this contribution to the systematic budget.

We construct our shear correlation function estimator following Kilbinger et al. (2013):

$\xi_{ \pm}(\theta)=\frac{\sum_{i, j} w_{i} w_{j}\left[e_{t}\left(\theta_{i}\right) e_{t}\left(\theta_{j}\right) \pm e_{r}\left(\theta_{i}\right) e_{r}\left(\theta_{j}\right)\right]}{\sum_{i, j} w_{i} w_{j}}$.

All galaxy pairs $(i, j)$ separated with angular distance $\left|\theta_{i}-\theta_{j}\right| \in \theta$ contribute to the same bin, with their contribution weighted by the product of their LENSFIT weights $w_{i} w_{j}$ (Miller et al. 2013). The shear quantities $e_{\mathrm{t}}$ and $e_{\mathrm{r}}$ are the tangential and cross-component of the galaxy ellipticity, measured in the coordinate system of the galaxy pair. We account for the shear calibration by measuring

$1+K(\theta)=\frac{\sum_{i, j} w_{i} w_{j}\left(1+m_{i}\right)\left(1+m_{j}\right)}{\sum_{i, j} w_{i} w_{j}}$

and dividing $\xi_{ \pm}$by $1+K$. As a rule of thumb, $K$ is $\sim-0.11$ at all angular scales, with variations smaller than 0.1 per cent. We finally exclude all pairs with $\theta<12$ arcsec in order to minimize contamination by post-stamp leakage across LENSFIT templates. We perform this measurement with ATHENA, ${ }^{17}$ and show our results in Fig. 3.

\subsection{Simulations}

In order to achieve a high-precision cosmic shear measurement, not only must the data be thoroughly tested for subtle systematics residuals, but the sampling variance must be accurately estimated, a quantity that is very hard to assess from the data. To overcome this difficulty, we rely on a suite of weak lensing simulations based on $W M A P 9+\mathrm{SN}+\mathrm{BAO}$ cosmology. As detailed in Harnois-Déraps $\&$ van Waerbeke (2015), the SLICS-LE suite consists of $60 \mathrm{deg}^{2}$ light cones extracted from 500 independent $N$-body realizations. The numerical weak lensing signal is precise to better than 10 per cent for $\xi_{+}$with $\theta>0.4 \operatorname{arcmin}$ (and $\theta>5 \operatorname{arcmin}$ for $\xi_{-}$). We construct the mock maps by combining the different redshift planes with a redshift source distribution that mimics that of the data. We then sample the simulated shear maps with $10^{5}$ points randomly located, and compute the shear two-point correlation functions $\xi_{ \pm}$ of these mock 'galaxies' with the same pipeline as the data (i.e. from equation 46).

\section{RESULTS}

In this section, we first review our error budget, we then describe how different components combine in our model rejection procedure, and finally present our results.

\subsection{Error budget}

This analysis closely follows that of HWVH; we summarize here the main ingredients, and refer the reader to the reference for more details. The sources of error in this analysis can be broken into three terms: (1) uncertainty on the cosmic shear measurement, (2) uncertainty in the theoretical model describing the non-linear regime of structure formation and (3) uncertainty on the fiducial cosmology.

(1) The error on our cosmic shear measurement is dominated by shape noise at small angles and sampling variance at large angles. The angular scales at which these two errors contribute equally occur at $\theta=2$ and 30 arcmin for $\xi_{+}$and $\xi_{-}$, respectively. In addition, the variance-shape noise mixed term contributes to about a third of the error on $\xi_{+}$at large angles, but is negligible in $\xi_{-}$, as seen in Kilbinger et al. (2013). We have estimated the sampling variance from the SLICS-LE weak lensing simulations, and added an extra contribution from the halo-sampling variance, following the modelling of Sato et al. (2009), which provides at most a 10 per cent correction on the overall error. Our measurement is minimally affected by intrinsic alignment of galaxies, since we do not perform tomographic analysis (see Heymans et al. 2013 for more details on intrinsic alignments in the CFHTLenS data). The error from shape reconstruction is already included in the statistical term, hence does

\footnotetext{
${ }^{17}$ ATHENA: http://cosmostat.org/athena.html
} 

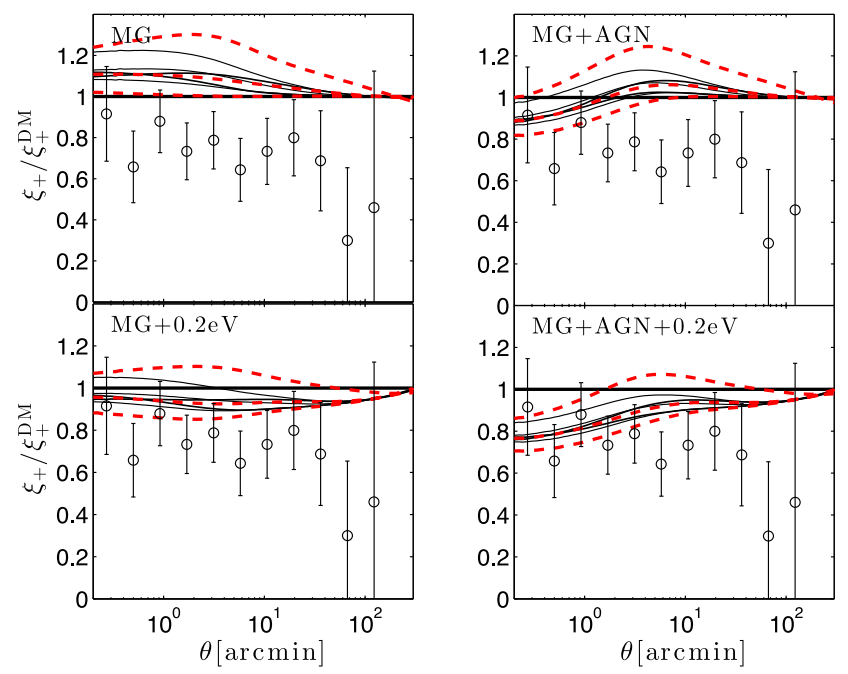

Figure 6. Same as Fig. 4, but for $\xi_{+}$. The open symbols represent our measurements from the CFHTLenS data, exactly as in Fig. 3. Shown are the $1 \sigma$ error bars.

not require an extra term. Photometric redshift uncertainty enters the measurement through modification of the source distribution $n(z)$, but this effect is negligible compared with other sources of error hence is not included.

(2) The uncertainty on the DM-ONLY non-linear model has been carefully assessed in HWVH by comparing five different predictions: HALOFIT2012, HALOFIT2011 + small scale empirical recalibration, Cosmic Emulator + power-law graft, Cosmic Emulator + HALOFIT 2012 graft and, finally, the mean over five independent highresolution simulations - the SLICS-HR suite described in HarnoisDéraps \& van Waerbeke (2015). These five models agree very well over most angular scales, and the $1 \sigma$ scatter among them is taken as the $\left(\theta\right.$-dependent) theoretical error. For $\xi_{+}$, angles larger than 5 arcmin achieve a 1 per cent precision, while smaller angles reach a 4 per cent precision. For $\xi_{-}$, angles larger than 3 arcmin have a 4 per cent precision, while smaller angles are only accurate to 8 per cent. The poorer precision on $\xi_{-}$is explained by the fact that it probes much deeper in the non-linear regime. Let us re-iterate that the precisions above mentioned represent the theoretical error on our weak lensing signal for a fixed $\Lambda \mathrm{CDM}$ cosmology universe, in which there is no modified gravity, baryonic feedback nor massive neutrinos. Also, we do not assign a theoretical error to the baryonic feedback model, but rather treat it as an exact effect that can be switched on and off (see Section 4.2). This is of course not exactly representative of the real Universe, and a full MCMC could be run on the parameters that describe the baryonic feedback, but leave this for a future study.

(3) The uncertainty in the cosmological parameters is set by the WMAP9 precision (Hinshaw et al. 2013), whose dominant contribution on the weak lensing uncertainty arises via the parameters $\Omega_{\mathrm{M}}$ and $A_{\mathrm{s}}$. With the inclusion of the BAO and SN external data, these two parameters are allowed a 3.4 and 3.3 per cent variation about their mean values $(1 \sigma)$. Since the amplitude of $\xi_{ \pm}$roughly scales as $\left(A_{\mathrm{s}} \Omega_{\mathrm{M}}\right)^{2}$, we expect the combined error to be of the order 5 per cent of the $\Lambda \mathrm{CDM}$ baseline signal, assuming no prior on the joint contour.

Note that the cosmological error and the modelling error (terms 2 and 3 ) enter in our analysis as systematic uncertainties, therefore we add them in quadrature and marginalize over them (see details in Section 4.2). Also note that the Planck cosmology $\left\{\Omega_{\mathrm{M}}, A_{\mathrm{s}}\right\}$ falls
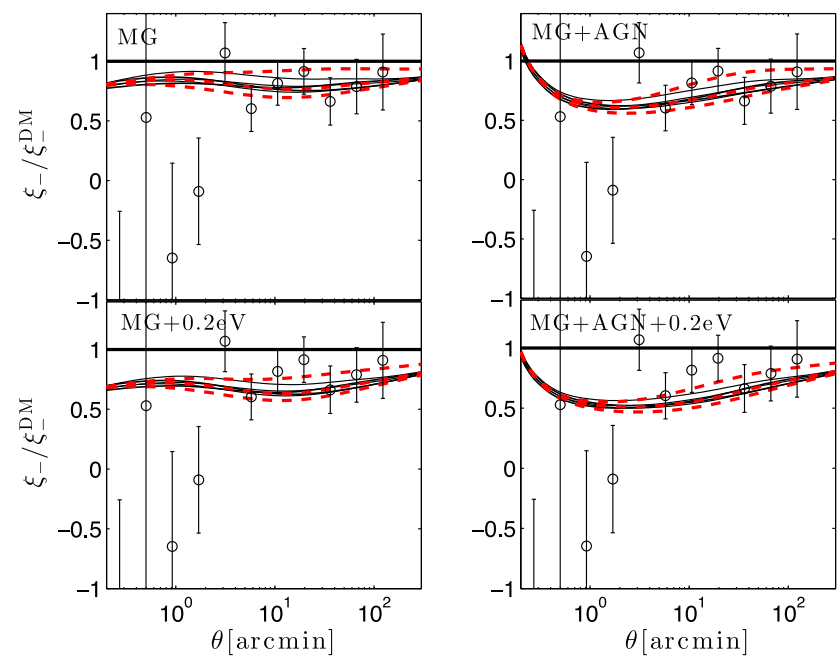

Figure 7. Same as Fig. 6, but for $\xi_{-}$. Note the different $y$-axis range compared to Fig. 6.

within our $3 \sigma$ search limits, although closer to the edge of the search zone. (See Figs 1 and 7 from HWMH for a quantitative assessment of these different sources of uncertainty.)

\subsection{Model rejection strategy}

As seen in Figs 6 and 7, the effects of baryons, massive neutrinos and modified gravity are significantly degenerate on $\xi_{ \pm}$. Given the noise levels in the current data and the number of internal parameters that describe these different mechanisms, performing a full MCMC analysis is not convenient to extract meaningful constraints. A more appropriate and direct way is to sample a finite set of model combinations and examine their agreement with the data. This case-by-case strategy has the potential to reject models that are inconsistent with the data, which can then be translated into constraints on the underlying free parameters.

The metric we adopt for this type of analysis is the $p$-value, which measures the probability that the data are consistent with the model, if the model is true. It is given by the integral over the $\chi^{2}$ probability density function, where the lower bound is the measured $\chi^{2}$ and the upper bound is infinity. As a rule of thumb, models with $p$-values $<10$ per cent are rejected with more than 90 per cent confidence, and $1 \sigma, 2 \sigma, 3 \sigma \ldots$ rejection measurements are obtained for $p$-values of $0.317,0.046,0.003 \ldots$ Our strategy therefore consists to measure the $\chi^{2}$ and $p$-value associated with each combination of baryon feedback, neutrino mass and modified gravity model, and to flag every combination with $p<0.1$ as being disfavoured.

The uncertainty arising from statistical and sampling variance naturally enters this calculation through the evaluation of the $\chi^{2}$, which involves the inversion of the cosmic shear covariance matrix. The systematic uncertainty, however, is trickier to capture. In our cosmic shear measurement, it mainly manifests itself as shifts in the amplitude of the signal, as described in Section 4.1. The systematic error is higher at smaller angles and represents at most an error of $\sim 9$ per cent on the $\xi_{ \pm}$model amplitude. In order to marginalize over this effect, for each model, we allow the theoretical signal to shift up and down by $3 \sigma_{\text {syst }}$, corresponding to vertical excursion of 27 percent in Figs 6 and 7, keeping the error bars (statistical + sampling) fixed. We then compute an array of $p$-values in this excursion range, and record only the largest measurement (i.e. the least restrictive). 
The exact number of degrees of freedom (d.o.f.) that enters the $\chi^{2}$ distribution function must be carefully chosen. To begin with, each of the two cosmic shear observables is organized in 11 angular bins, yielding a maximum of 22 d.o.f. However, assigning one d.o.f. per angular bin would be incorrect, for the following reason. In a statistical sense, our model rejection method is completely equivalent to fitting the parameter combination $\left(A_{\mathrm{s}}^{2} \Omega_{\mathrm{M}}^{1.8}\right)$ from the amplitude of the $\xi_{ \pm}$signals, followed by an extraction of the most likely neutrino mass for each baryon feedback and modified gravity model from the largest $p$-value. This implies that the number of degrees of freedom should be reduced by two (one for fitting $A_{\mathrm{s}}^{2} \Omega_{\mathrm{M}}^{1.8}$, one for fitting $M_{v}$ ) in the conversion between $\chi^{2}$ and $p$-values.

Note that for a given angular scale, both $\xi_{+}$and $\xi_{-}$probe different physical scales, the latter focusing on structures about five times smaller. It is therefore relevant to examine the constraining power of $\xi_{+}$first, and to add $\xi_{-}$to the data vector as a second step. When both are combined, the full data covariance matrix involves the cross-correlation region, as described in HWVH. The resulting $p$-values are summarized for all our results in Table 2, for $M_{v} \leq 0.2 \mathrm{eV}$. No conclusions can be drawn from models with higher total neutrino masses, as the $p$-values for any combination is always greater than 0.175 . The models rejected at more than $1.64 \sigma$ (i.e. 90 per cent confidence interval, CI) are highlighted in bold font.

\subsection{Discussion}

One of the main results recovered from Table 2 is that the $f(R)$ model with $\left\{\left|f_{R_{0}}\right|, n\right\}=\left\{10^{-4}, 1\right\}$ is strongly disfavoured by the cosmic shear data, regardless of the baryonic feedback model or sum of neutrino mass, consistent with independent constraints. The $f(R)$ and $f(R)+$ AGN models are rejected by at least $3 \sigma$, but combinations including massive $(0.2 \mathrm{eV})$ neutrinos tend to weaken these constraints. This can be understood by the fact that massive neutrinos and modified gravity partly compensate for one another, reducing the global departure from $\Lambda$ CDM. Also, $f(R, n=2)$ models are generally in better agreement with the data compared to their $(n=1)$ counterpart. This is so simply because higher values of $n$ rapidly suppress the $f(R)$ term, hence deviations from GR, as seen in equation (22).

The next important result is that the rejection of massless neutrinos + DM-ONLY is robust against all modified gravity models we have tested, and typically made stronger. The cosmic shear data clearly prefer lower values of $\xi_{ \pm}$at small angular scales, and modified gravity pulls the other way. Also, 9 out of the 21 modified gravity models are still consistent with the $\xi_{+}$data alone.

The inclusion of baryon feedback reduces to about two-thirds the number of models rejected with 90 per cent CI. For instance, dilation models A2, A3, B3, B4, C1, C3, D1, E3 and E4 are disfavoured; these are the most discrepant with GR $+\Lambda \mathrm{CDM}$. Referring to Table 1 and the model descriptions in Section 2.1, this can be interpreted as follow. In a tomographic parametrization of modified gravity centred on $\left\{m_{0}, r, \beta_{0}, s\right\}=\{0.334,1.0,0.5,0.24\}$, excursions in the $s, \beta_{0}$ and $r$ directions are studied with models $\mathrm{A}, \mathrm{B}$ and $\mathrm{C}$, respectively, and the data favour lower parameters values. Model $\mathrm{E}$ explores the $m_{0}$ direction, and the data prefer higher values. Model $\mathrm{D}$ explores the diagonal direction in the $\left\{m_{0}, s\right\}$ plane at fixed $A_{2}$ (see equation 8 ), where here we observe instead that the data prefer lower $m_{0}$ values.

In comparison with the combined $\xi_{+} \xi_{-}$vector, the $\xi_{+}$data vector provides a qualitatively similar result, except with a rejection power a bit lowered. There is thus a net gain in using the combined vector, even though the smallest angular scales of $\xi_{\text {- }}$ are heavily downweighted due to theoretical uncertainties.

We note that there is a mild effect seen in the 'AGN' column

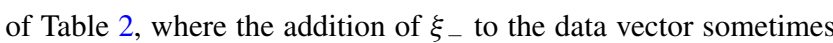
increases the $p$-value by a small amount. This can be attributed to the fact that at small angles, $\xi$ - prefers amplitude even lower than $\xi_{+}$, compared to the DM-ONLY model. Adding baryon feedback therefore produces a lower rejection rate in the former than in the latter quantity.

When neutrino masses are allowed to reach $0.2 \mathrm{eV}$, only the $f(R)\left\{10^{-4}, 1\right\}, f(R)\left\{10^{-4}, 2\right\}$ and the Dilaton B4 and E4 models remain in tension with the data. With $\mathrm{AGN}+M_{v}=0.2 \mathrm{eV}$, no models are rejected, aside from the most extreme case considered in this paper: $f(R)\left\{10^{-4}, 1\right\}$.

This means that given the current cosmic shear data and levels of systematics, it is possible to accommodate most models, as long as either massive neutrinos or strong baryon feedback mechanisms counter-balance the effect of the fifth force on the matter clustering. As upcoming independent cosmological probes will tighten the uncertainty on neutrino masses and significantly improve the statistical and sampling errors, we expect the next generation of such analysis to be much more constraining. Once at this stage, it will be instructive to propagate our measurements on to $\{m(a), \beta(a)\}$ contours and provide a Fisher matrix for joint probes analyses. If, for instance, the total mass turns out to be much smaller than $0.2 \mathrm{eV}$, then the current AGN column should give a very good approximation of the rejection power from the CFHTLenS cosmic shear data. Precise modelling of the baryon feedback is likely to take more time to reach, due to the higher level of complexity intrinsic to these astrophysical phenomena. Intermediate solutions will involve a series of tuneable parameters, also to be constrained.

On a separate note, we stress that the constraints can be further tightened using additional information about the weak-lensing observables, such as the non-Gaussian features (Munshi et al. 2012), or by combining the results with external probes such as redshift distortions, peculiar velocity, etc.

\section{CONCLUSION}

Cosmic shear is a promising tool for probing deviations from GR, since these are maximal at scales of a few Mpc, where the lensing signal-to-noise ratio is the highest. These same scales are very challenging to probe with other types of large-scale structure observables, mainly because of the galaxy bias that is largely unknown. At the same time, this complementarity offers a number of opportunities for strong constraints based on joint data sets.

One of the main challenges in working with these non-linear scales is the large theoretical uncertainties due to the unknown neutrino masses, the precise baryonic feedback mechanisms and, to a lesser extent, inaccuracies in the clustering of dark matter. However, a lot of effort is invested in all these areas, such that it becomes possible to place joint constraints on these degenerate physical effects.

This paper presents the first constraints on modified gravity obtained from cosmic shear measurements alone; the results are derived by studying the impact of modified gravity on matter clustering and comparing the predictions with the public CFHTLenS data. Limiting the background $\Lambda \mathrm{CDM}$ cosmology to the $3 \sigma$ range in $\left\{A_{\mathrm{s}}, \Omega_{\mathrm{M}}\right\}$ allowed by $W M A P 9+\mathrm{SN}+\mathrm{BAO}$, we compared the $\xi_{ \pm}$data against predictions including $f(R)$ and Dilaton models in a number of parameter configurations. We carried a model rejection analysis accounting for possible degeneracies with massive 
Table 2. Distribution of $p$-values for different combinations of baryon feedback models, neutrino masses and gravity models (see main text for details). The parameters listed in the leftmost column of the $f(R)$ models are $\left\{n,\left|f_{R_{0}}\right|\right\}$. Dilaton models are described in Table 1. For this calculation, we fit all data in the range $0.2<\theta<$ 167 arcmin. Specifically, each entry in this table represents the largest $p$-value probed inside a $3 \sigma_{\text {syst }}$ region about the mean of the model. Values in bold face highlight the model combinations that are excluded by the data with more than $1.64 \sigma$ significance ( $p$-value $<0.1$, equivalent to a CI of 90 per cent). Models with $M_{v}>0.2 \mathrm{eV}$ are not listed, as none has value lower than 0.176 .

\begin{tabular}{|c|c|c|c|c|c|c|c|c|}
\hline \multirow[b]{2}{*}{ Model } & \multicolumn{2}{|c|}{ DM-ONLY } & \multicolumn{2}{|c|}{ AGN } & \multicolumn{2}{|c|}{$0.2 \mathrm{eV}$} & \multicolumn{2}{|c|}{$\mathrm{AGN}+0.2 \mathrm{eV}$} \\
\hline & $\xi_{+}$ & $\xi_{+} \xi_{-}$ & $\xi_{+}$ & $\xi_{+} \xi_{-}$ & $\xi_{+}$ & $\xi_{+} \xi_{-}$ & $\xi_{+}$ & $\xi_{+} \xi_{-}$ \\
\hline \multicolumn{9}{|c|}{ GR } \\
\hline$\Lambda \mathrm{CDM}$ & 0.132 & 0.065 & 0.119 & 0.150 & 0.331 & 0.297 & 0.289 & 0.444 \\
\hline \multicolumn{9}{|c|}{ Generalized Dilaton } \\
\hline $\mathrm{A} 1$ & 0.126 & 0.058 & 0.116 & 0.141 & 0.323 & 0.282 & 0.284 & 0.431 \\
\hline A2 & 0.088 & 0.030 & 0.093 & 0.099 & 0.269 & 0.203 & 0.256 & 0.363 \\
\hline $\mathrm{A} 3$ & 0.037 & 0.008 & 0.060 & 0.051 & 0.184 & 0.105 & 0.207 & 0.265 \\
\hline $\mathrm{B} 1$ & 0.120 & 0.054 & 0.113 & 0.135 & 0.315 & 0.273 & 0.281 & 0.424 \\
\hline B3 & 0.043 & 0.010 & 0.064 & 0.054 & 0.195 & 0.113 & 0.212 & 0.272 \\
\hline B4 & 0.008 & 0.001 & 0.032 & 0.019 & 0.107 & 0.037 & 0.150 & 0.161 \\
\hline $\mathrm{C} 1$ & 0.080 & 0.022 & 0.100 & 0.098 & 0.259 & 0.171 & 0.271 & 0.365 \\
\hline $\mathrm{C} 3$ & 0.104 & 0.040 & 0.098 & 0.111 & 0.293 & 0.239 & 0.261 & 0.385 \\
\hline $\mathrm{C} 4$ & 0.114 & 0.049 & 0.104 & 0.122 & 0.307 & 0.259 & 0.267 & 0.402 \\
\hline D1 & 0.063 & 0.013 & 0.100 & 0.085 & 0.232 & 0.132 & 0.276 & 0.347 \\
\hline D3 & 0.127 & 0.060 & 0.115 & 0.141 & 0.325 & 0.286 & 0.282 & 0.431 \\
\hline D4 & 0.131 & 0.064 & 0.119 & 0.149 & 0.331 & 0.295 & 0.288 & 0.441 \\
\hline E1 & 0.118 & 0.049 & 0.117 & 0.135 & 0.314 & 0.260 & 0.289 & 0.425 \\
\hline E3 & 0.047 & 0.013 & 0.053 & 0.049 & 0.200 & 0.132 & 0.186 & 0.257 \\
\hline E4 & 0.026 & 0.007 & 0.032 & 0.027 & 0.156 & 0.094 & 0.138 & 0.188 \\
\hline \multicolumn{9}{|c|}{$f(R)$} \\
\hline$\left\{1,10^{-4}\right\}$ & 0.001 & 0.000 & 0.005 & 0.003 & 0.051 & 0.018 & 0.054 & 0.057 \\
\hline$\left\{1,10^{-5}\right\}$ & 0.058 & 0.013 & 0.072 & 0.062 & 0.222 & 0.134 & 0.222 & 0.292 \\
\hline$\left\{1,10^{-6}\right\}$ & 0.129 & 0.054 & 0.125 & 0.145 & 0.328 & 0.271 & 0.298 & 0.437 \\
\hline$\left\{2,10^{-4}\right\}$ & 0.011 & 0.003 & 0.020 & 0.014 & 0.112 & 0.056 & 0.104 & 0.131 \\
\hline$\left\{2,10^{-5}\right\}$ & 0.095 & 0.030 & 0.094 & 0.097 & 0.277 & 0.200 & 0.254 & 0.354 \\
\hline$\left\{2,10^{-6}\right\}$ & 0.137 & 0.063 & 0.126 & 0.154 & 0.338 & 0.292 & 0.299 & 0.449 \\
\hline
\end{tabular}

neutrinos and baryonic feedback mechanisms, and investigated which combinations of models were mostly disfavoured by the data. As summarized in Table 2, the $f(R)$ model with $\left|f_{R_{0}}\right|=10^{-4}$ is strongly disfavoured even in the presence of realistic levels of baryonic feedback and massive neutrinos reaching $M_{v}=0.2 \mathrm{eV}$. A universe with no baryonic feedback and massless neutrinos is also rejected with $2 \sigma$ or above in most modified gravity scenarios. We are not yet able to identify a preferred model with the current level of statistical accuracy, but we expect future weak lensing experiments to improve significantly in this direction.

In our analyses, we have used the simplifying assumption that the biases due to massive neutrinos, baryon feedback and modified gravity were uncorrelated, which is justified to some extend based on the several numerical results. However, precise correlations will need to be studied for a number of models, a task that involves suites of large cosmological hydrodynamical simulations including all the ingredients at once.

One important future task will be to map observational detections of modifications to GR on to parameter constraints such as the $\{m(a), \beta(a)\}$ pair. However, the current data are not quite there yet. Several theories can accommodate similar phenomenological effects, and model-independent parameterizations such as that presented in Leonard, Baker \& Ferreira (2015) might prove helpful for this.

This paper used the impact of modified gravity on the clustering properties of matter and their propagation on to the weak lensing cosmic shear signal. Other avenues of probing deviations from GR with weak lensing data are complementary, including direct combinations with baryonic probes or tomographic decomposition, and worth exploring in the near future.

\section{ACKNOWLEDGEMENTS}

We would like to thank Alexander Mead for his comments on the manuscript, and to acknowledge useful discussions with Alexei Starobinsky, Alan Heavens, Catherine Heymans and Massimo Viola. We are deeply grateful to all the CFHTLenS team for having made public their high-quality shear data. Computations for the $\mathrm{N}$-body simulations were performed on the GPC supercomputer at the SciNet HPC Consortium. SciNet is funded by: the Canada Foundation for Innovation under the auspices of Compute Canada; the Government of Ontario; Ontario Research Fund - Research Excellence; and the University of Toronto. This work is based on observations obtained with MegaPrime/MegaCam, a joint project of CFHT and CEA/IRFU, at the Canada-France-Hawaii Telescope (CFHT) which is operated by the National Research Council (NRC) of Canada, the Institut National des Sciences de l'Univers of the Centre National de la Recherche Scientifique (CNRS) of France, and the University of Hawaii. This research used the facilities of the Canadian Astronomy Data Centre operated by the National Research Council of Canada with the support of the Canadian Space Agency. CFHTLenS data processing was made possible thanks to significant computing support from the NSERC Research Tools and Instruments grant program. JHD and LvW are funded by the 
NSERC and Canadian Institute for Advanced Research CIfAR; DM and PC acknowledge support from the Science and Technology Facilities Council (grant numbers ST/L000652/1; LR and PV receive support from the French Agence Nationale de la Recherche under Grant ANR-12-BS05-0002; PB acknowledges partial support from the European Union FP7 ITN INVISIBLES (Marie Curie Actions, PITN-GA-2011-289442) and from the Agence Nationale de la Recherche under contract ANR 2010 BLANC 041301 .

\section{REFERENCES}

Asaba S., Hikage C., Koyama K., Zhao G.-B., Hojjati A., Pogosian L., 2013, J. Cosmol. Astropart. Phys., 08, 029

Baldi M., Villaescusa-Navarro F., Viel M., Puchwein E., Springel V., Moscardini L., 2014, MNRAS, 440, 75

Bean R., Bernat D., Pogosian L., Silvestri A., Trodden M., 2007, Phys. Rev. D, 75, 064020

Benjamin J. et al., 2013, MNRAS, 431, 1547

Bernardeau F., Colombi S., Gaztanaga E., Scoccimarro R., 2002, Phys. Rep., 367,1

Bertschinger E., 2006, ApJ, 648, 797

Bird S., Viel M., Haehnelt M. G., 2012, MNRAS, 420, 2551

Brax P. C. van de Bruck C., Davis A. C., Shaw D. J., 2008, Phys. Rev. D, 78,104021

Brax P., Valageas P., 2013, Phys. Rev. D, 88, 023527

Brax P., Valageas P., 2014a, Phys. Rev. D, 90, 023507

Brax P., Valageas P., 2014b, Phys. Rev. D, 90, 023508

Brax P., van de Bruck C., Davis A.-C., Li B., Schmauch B., Shaw D.J., 2011 , Phys. Rev. D, 84, 123524

Brax P., Davis A.-C., Li B., Winther H. A., Zhao G.-B., 2012a, J. Cosmol. Astropart. Phys., 10, 002

Brax P., Davis A.-C., Li B., 2012b, Phys. Rev. D, 86, 044015

Brax P., Davis A.-C., Li B., 2012c, Phys. Lett. B, 715, 38

Buchdahl H. A., 1970, MNRAS, 150, 1

Carloni S., Dunsby P., Troisi A., 2008, Phys. Rev. D, 77, 024024

Chiba T., 2003, Phys. Lett. B, 575, 1

Clifton T., Ferreira P. G., Padilla A., Skordis C., 2012, Phys. Rep., 513, 1

Damour T., Polyakov A. M., 1994, Nucl. Phys. B, 423, 532

Erben T. et al., 2013, MNRAS, 433, 2545

Guzzo L. et al., 2008, Nature, 451, 541

Hall A., Bonvin C., Challinor A., 2013, Phys. Rev. D, 87, 064026

Hammami A., Llinares C., Mota D. F., Winther H. A., 2015, 449, 3635

Harnois-Déraps J., van Waerbeke L., 2015, MNRAS, 450, 2857

Harnois-Déraps J., van Waerbeke L., Viola M., Heymans C., 2015, MNRAS, 450, $1212(\mathrm{HWVH})$

Heavens A. F., Kitching T. D., Verde L., 2007, MNRAS, 380, 1029

Heitmann K., Lawrence E., Kwan J., Habib S., Higdon D., 2014, ApJ, 780, 111

Heymans C. et al., 2012, MNRAS, 427, 146

Heymans C. et al., 2013, MNRAS, 432, 2433

Hildebrandt H. et al., 2012, MNRAS, 421, 2355

Hinshaw G. et al., 2013, ApJ, 208, 19

Hinterbichler K., Khoury J., 2010, Phys. Rev. Lett., 104, 231301

Hu W., Sawicki I., 2007, Phys. Rev. D, 76, 064004

Jain B., Zhang P., 2008, Phys. Rev. D, 78, 063503

Jennings E., Baugh C. M., Li B., Zhao G.-B., Koyama K., 2012, MNRAS, 425,2128

Johnson A. et al., 2014, MNRAS, 444, 3926

Johnson A., Blake C., Dossett J., Koda J., Parkinson D., Joudaki S., 2015, preprint (arXiv:1504.06885)

Joyce A., Jain B., Khoury J., Trodden M., 2015, Phys. Rep., 568, 1

Kilbinger M. et al., 2013, MNRAS, 430, 2200

Koivisto T., 2006, Phys. Rev. D, 73, 083517

Koyama K., Taruya A., Hiramatsu T., 2009, Phys. Rev. D, 79, 123512

Leonard D., Baker T., Ferreira P. G., 2015, Phys. Rev. D, 91, 083504

Lewis A., Challinor A., Lasenby A., 2000, ApJ, 538, 473
Li Y., Hu W., 2011, Phys. Rev. D, 84, 084033

Li B., Zhao G.-B., Koyama K., 2012, MNRAS, 421, 3481

Li B., Hellwing W. A., Koyama K., Zhao G.-B., Jennings E., Baugh C. M., 2013a, MNRAS, 428, 743

Li B., Barreira A., Baugh C. M., Hellwing W. A., Koyama K., Pascoli S., Zhao G.-B., 2013b, J. Cosmol. Astropart. Phys., 1311, 012

Llinares C., Mota D. F., 2013, Phys. Rev. Lett., 110, 151104

Lombriser L., Slosar A., Seljak U., Hu W., 2010, Phys. Rev. D, 85, 124038

Lombriser L., Koyama K., Zhao G.-B., Li B., 2012, Phys. Rev. D, 85, 124054

Lombriser L., Li B., Koyama K., Zhao G.-B., 2013, Phys. Rev. D, 87, 123511

MacCrann N., Zuntz J., Bridle S., Jain B., Becker M. R., 2014, MNRAS, 451, 2877

Miller L. et al., 2013, MNRAS, 429, 2858

Motohashi H., Starobinsky A., Yokoyama J., 2009, Int. J. Mod. Phys. D, 18, 1731

Munshi D., van Waerbeke L., Smidt J., Coles P., 2012, MNRAS, 419, 536

Natarajan A., Zentner A. R., Battaglia N., Trac H., 2014, Phys. Rev. D, 90, 063516

Nunez A., Solganik S., 2004, preprint (arXiv:hep-th/0403159)

Olive K. A., Pospelov M., 2008, Phys. Rev. D, 77, 043524

Oyaizu H., Lima M., Hu W., 2008, Phys. Rev. D, 78, 123524

Perlmutter S. et al., 1999, ApJ, 517, 565

Pietroni M., 2005, Phys. Rev. D, 72, 043535

Planck Collaboration XIII, 2015, preprint (arXiv:1502.01589)

Planck Collaboration XIV, 2015, preprint (arXiv:1502.01590)

Pogosian L., Silvestri A., 2008, Phys. Rev. D, 77, 023503

Riess A. G. et al., 1998, AJ, 116, 1009

Sato M., Hamana T., Takahashi R., Takada M., Yoshida N., Matsubara T., Sugiyama N., 2009, ApJ, 701, 945

Schaye J. et al., 2010, MNRAS, 402, 1536

Schimdt F., 2008, Phys. Rev. D, 78, 3002

Semboloni E., Hoekstra H., Schaye J., van Daalen M. P., McCarthy I. G., 2011, MNRAS, 417, 2020

Simpson F. et al., 2013, MNRAS, 429, 2249

Song Y.-S., Hu W., Sawicki I., 2007a, Phys. Rev. D, 75, 044004

Song Y.-S., Peiris H., Hu W., 2007b, Phys. Rev. D, 76, 063517

Starobinsky A. A., 1980, Phys. Lett. B, 91, 99

Starobinsky A. A., 2007, JETP Lett., 86, 157

Taddei L., Catena R., Pietroni M., 2014, Phys. Rev. D, 89, 023523

Takahashi R., Sato M., Nishimichi T., Taruya A., Oguri M., 2012, ApJ, 761, 152

Tsujikawa S., 2007, Phys. Rev. D, 76, 023514

Tsujikawa S., Tatekawa T., 2008, Phys. Lett. B, 665, 325

Valageas P., 2013, Phys. Rev. D, 88, 083524

Valageas P., Nishimichi T., 2011, A\&A, 527, A87

Valageas P., Nishimichi T., Taruya A., 2013, Phys. Rev. D, 87, 083522

van Daalen M. P., Schaye J., Booth C. M., Dalla Vecchia C., 2011, MNRAS, 415,3649

Wilcox H. et al., 2015, MNRAS, 452, 1171

Zhang P., 2006, Phys. Rev. D, 73, 123504

Zhao G.-B., 2014, ApJS, 211, 23

\section{APPENDIX A: DETAILS ON THE THEORETICAL MODELLING OF $P(k)$ IN MODIFIED GRAVITY SCENARIOS}

This appendix discusses the construction strategy of the matter density power spectrum $P(k ; z)$ in the presence of $f(R)$ or Dilaton modifications to GR; full details are provided in the references contained herein.

\section{A1 Two-halo term: $P_{2 \mathrm{H}}(k)$}

The power spectrum analytical prediction is constructed from a halo model approach, following equation (42). The two-halo term, which dominates on large scales, is computed from a 
Lagrangian-space resummation of standard perturbation theory that is exact up to order $P_{\mathrm{L}}^{2}$ and contains partial resummations of higher order terms. It is also supplemented with non-perturbative contributions that take into account some aspects of shell crossing and ensure that all particle pairs are counted only once in the sum. Within this framework, the large-scale term $P_{2 \mathrm{H}}(k)$ essentially contains no free parameter. It can therefore be computed in $\Lambda \mathrm{CDM}$ and modified-gravity scenarios by using perturbation theory up to order $P_{\mathrm{L}}^{2}$ (which requires going to order $\delta_{\mathrm{L}}^{3}$ in terms of the density field itself).

In the case of the $\Lambda \mathrm{CDM}$ cosmology, this perturbative expansion follows the standard approach (Bernardeau et al. 2002), where the density and velocity fields are written as perturbative expansions over powers of the linear density field $\delta_{\mathrm{L}}$; subsequent orders are computed by substituting into the continuity and EulerPoisson equations. In the case of the modified-gravity scenarios considered in this paper, we follow the same approach but require an additional expansion to write the fifth force in terms of the non-linear density fluctuations. Indeed, using the quasi-static approximation, we can relate the scalar field $\varphi$ to the matter density field $\rho$, typically through a non-linear Klein-Gordon equation. Then, we can solve for $\varphi$ as an expansion over the non-linear density fluctuations $\delta \rho$. This allows us to obtain both the Newtonian potential and the fifth-force potential as functionals of the nonlinear matter density fluctuations. However, while the Newtonian potential is given by the linear Poisson equation, the fifth-force potential is usually given by a non-linear equation that involves new time and scale dependences. In terms of the diagrammatic expansion of the non-linear power spectrum $P(k)$ over $P_{\mathrm{L}}(k)$, this implies that the linear propagators and the vertices are modified with new diagrams associated with the new non-linearity of the modified Poisson equation, see Brax \& Valageas (2013) for more explanations.

\section{A2 One-halo term: $\boldsymbol{P}_{1 \mathrm{H}}(\boldsymbol{k})$}

The one-halo term is obtained from the halo mass function and the halo density profile, with the addition of a counter-term first introduced in Valageas \& Nishimichi (2011) that arises from mass conservation. This also ensures that $P_{1 \mathrm{H}}(k)$ decays at low $k$ and becomes subdominant as compared with $P_{2 \mathrm{H}}(k)$, whereas the usual formulation gives a spurious white-noise tail that dominates on very large scales. We take into account the impact of modified gravity through its effect on the halo mass function (i.e. through the acceleration or slowing down of the spherical collapse), but neglect the impact on the halo shape and profile. This should be sufficient for our purposes, because we only consider cosmologies that re- main close to the $\Lambda \mathrm{CDM}$ reference, and these modified gravity models have a much stronger impact on the halo mass function, especially on its large-mass tail, than on the halo profile. As shown in Valageas (2013), at $z=0$, a 10 percent change to the massconcentration relation only yields a 2 percent change of $P(k)$ at

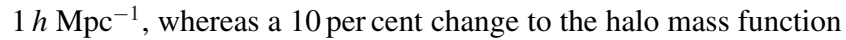
yields a 2 per cent change of $P(k)$ at $0.35 h \mathrm{Mpc}^{-1}$ and a 7.5 per cent change at $1 h \mathrm{Mpc}^{-1}$. Generally, the concentration parameter always remains in the range 3-10 for typical haloes and does not vary by much more than 10 per cent for realistic scenarios, whereas the mass function at $M \sim 5 \times 10^{14} h^{-1} \mathrm{M}_{\odot}$ can vary by more than 50 per cent (Lombriser et al. 2012, 2013). The interior of haloes is mostly affected by screening anyway, further justifying this approximation.

\section{A3 Comparison with numerical simulations}

The modelling described above for the matter density power spectrum has been checked in details against numerical simulations in Valageas et al. (2013) for $\Lambda \mathrm{CDM}$ cosmologies, and in Brax \& Valageas (2013) for the class of modified gravity models that we consider in this paper. In the case of $\Lambda \mathrm{CDM}$, it provides an accuracy of 2 percent up to comoving wavenumber $k \sim 0.9 \mathrm{~h} \mathrm{Mpc}^{-1}$, and 15 per cent up to $k=15 \mathrm{~h} \mathrm{Mpc}^{-1}$ down to $z=0.35$. In terms of the real-space correlation function, this translates into an accuracy of 5 per cent down to the comoving scale $r=0.15 h^{-1} \mathrm{Mpc}$. For the $f(R)$ theories, it is able to reproduce very well the deviations from the $\Lambda \mathrm{CDM}$ scenarios up to $k=3 \mathrm{~h} \mathrm{Mpc}^{-1}$ (the highest wavenumber available from the simulations) at $z=0$, for $\left|f_{R_{0}}\right|=10^{-4}, 10^{-5}$, and $10^{-6}$. In particular, it accurately captures the relative effect on the power compared to the $\Lambda \mathrm{CDM}$ reference due to the non-linear Chameleon mechanism. For the Dilaton models, the agreement with the numerical simulations depends somewhat on the model parameters but it typically gives a good quantitative estimate of the deviations from $\Lambda \mathrm{CDM}$ up to $k=5 \mathrm{~h} \mathrm{Mpc}^{-1}$ (the highest wavenumber available from the simulations). When there is a noticeable departure from the simulations, it corresponds to an underestimation of the amplification of the power spectrum at $k \gtrsim 2 \mathrm{~h} \mathrm{Mpc}^{-1}$, which may be due to our neglect of the impact of modified gravity on the halo concentration parameter. Therefore, in such cases our approach provides a conservative estimate of the deviations from $\Lambda \mathrm{CDM}$. Again, this modelling is able to capture the decrease of the deviations from the $\Lambda \mathrm{CDM}$ reference due to the non-linear Damour-Polyakov mechanism.

This paper has been typeset from a $\mathrm{T}_{\mathrm{E}} \mathrm{X} / \mathrm{LT} \mathrm{E} \mathrm{X}$ file prepared by the author. 